\title{
Displacement of oil by water in a single elastic capillary
}

\author{
AM Meirmanov ${ }^{1}$, OV Galtsev ${ }^{2 *}$ and RN Zimin ${ }^{3}$
}

\section{"Correspondence:}

oleg_galtsev@mail.ru

${ }^{2}$ Belgorod National Research

University, Pobedi 85 , Belgorod,

308015, Russia

Full list of author information is

available at the end of the article

\begin{abstract}
In this paper we consider the evolution of a free boundary separating two immiscible viscous fluids with different constant densities. The joint motion of liquids in the solid skeleton is described by Stokes equations coupled with Lamé equations, driven by the input pressure and the force of gravity.

We prove the existence and uniqueness of classical solutions global in time, and we emphasize the study of the properties of the moving boundary separating the two fluids.
\end{abstract}

Keywords: free boundary problems; Stokes and Lamé equations; Muskat problem

\section{Introduction}

This paper follows our previous paper [1], where we considered the evolution of a free boundary separating two immiscible viscous fluids in a single capillary of an absolutely rigid solid body. Here we consider the same flow in a single capillary $Q_{f} \subset Q$ of an elastic solid body.

Suppose for simplicity that

$$
Q=\left\{\mathbf{x} \in \mathbb{R}^{2}:-1<x_{i}<1, i=1,2\right\}, \quad Q_{f}=\left\{\mathbf{x}:-1<x_{1}<1,-\frac{1}{2}<x_{2}<\frac{1}{2}\right\} .
$$

In dimensionless variables the evolution of a flow is driven by the input pressure and the force of gravity. More precisely, in this problem we have to find the velocity $\mathbf{u}^{f}(\mathbf{x}, t)$, the pressure $p_{f}(\mathbf{x}, t)$ and the density $\rho_{f}(\mathbf{x}, t)$ of the non-homogeneous liquid in $Q_{f}$, and the displacements $\mathbf{u}^{s}(\mathbf{x}, t)$ and the pressure $p_{s}(\mathbf{x}, t)$ of the elastic skeleton in $Q_{s}=Q \backslash \overline{Q_{f}}$ from the following system of differential equations:

$$
\begin{aligned}
& \nabla \cdot \mathbb{P}_{f}+\rho_{f} \mathbf{e}=0, \quad \nabla \cdot \mathbf{u}^{f}=0, \quad \mathbf{x} \in Q_{f}, 0<t<T, \\
& \nabla \cdot \mathbb{P}_{s}+\rho_{s} \mathbf{e}=0, \quad \nabla \cdot \mathbf{u}^{s}=0, \quad \mathbf{x} \in Q_{s}, 0<t<T, \\
& \frac{d \rho_{f}}{d t} \equiv \frac{\partial \rho_{f}}{\partial t}+\nabla \cdot\left(\rho_{f} \mathbf{u}^{f}\right)=\frac{\partial \rho_{f}}{\partial t}+\mathbf{u}^{f} \cdot \nabla \rho_{f}=0, \quad \mathbf{x} \in Q_{f}, 0<t<T,
\end{aligned}
$$

where

$$
\mathbb{P}_{f}=2 \mu \mathbb{D}\left(\mathbf{u}^{f}\right)-p_{f} \mathbb{I}, \quad \mathbb{D}\left(\mathbf{u}^{f}\right)=\frac{1}{2}\left(\nabla \mathbf{u}^{f}+\left(\nabla \mathbf{u}^{f}\right)^{*}\right),
$$

(0) The Author(s) 2017. This article is distributed under the terms of the Creative Commons Attribution 4.0 International License (http://creativecommons.org/licenses/by/4.0/), which permits unrestricted use, distribution, and reproduction in any medium, provided you give appropriate credit to the original author(s) and the source, provide a link to the Creative Commons license, and indicate if changes were made. 


$$
\mathbb{P}_{s}=2 \lambda \mathbb{D}\left(\mathbf{u}^{s}\right)-p_{s} \mathbb{I}
$$

$\mu=$ const is the viscosity of the liquids, $\lambda=$ const is the Lamé coefficient, $\mathbf{e}$ is the given vector, $\rho_{s}$ is the density of the solid body, and $\mathbb{I}$ is the unit tensor.

The mass and momentum conservation laws dictate the coincidence of velocities and normal tensions in the liquid and solid components,

$$
\mathbf{u}^{f}=\frac{\partial \mathbf{u}^{s}}{\partial t}, \quad \mathbb{P}_{f} \cdot \mathbf{n}=\mathbb{P}_{s} \cdot \mathbf{n},
$$

on the common boundary $S=\partial Q_{f} \cap \partial Q_{s}$ with unit normal vector $\mathbf{n}$.

The boundary condition on the lateral part $S^{0}=\left\{x_{2}= \pm 1\right\}$ of the boundary $\partial Q$ for $0<$ $t<T$ has the form

$$
\mathbf{u}^{s}(\mathbf{x}, t)=0
$$

At the 'entrance' and 'exit' boundaries $S^{ \pm}=\left\{\mathbf{x} \in \partial Q: x_{1}=\mp 1\right\}$

$$
\begin{aligned}
& \mathbb{P}_{s} \cdot \mathbf{e}_{1}=-p^{+}(\mathbf{x}) \mathbf{e}_{1}, \quad \mathbf{x} \in S_{s}^{+}, \quad \mathbb{P}_{f} \cdot \mathbf{e}_{1}=-p^{+}(\mathbf{x}) \mathbf{e}_{1}, \quad \mathbf{x} \in S_{f}^{+}, 0<t<T, \\
& \mathbb{P}_{s} \cdot \mathbf{e}_{1}=0, \quad \mathbf{x} \in S_{s}^{-}, \quad \mathbb{P}_{f} \cdot \mathbf{e}_{1}=0, \quad \mathbf{x} \in S_{f}^{-}, 0<t<T,
\end{aligned}
$$

where $p^{+}(\mathbf{x})$ is a given function, $S_{f}^{ \pm}=S^{ \pm} \cap \partial Q_{f}, S_{s}^{ \pm}=S^{ \pm} \cap \partial Q_{s}$, and $\mathbf{e}_{i}$ is the unit vector of the $x_{i}$-axis for $i=1,2$.

To simplify our considerations we pass to the homogeneous boundary conditions at $S^{ \pm}$,

$$
\mathbb{P}_{i} \cdot \mathbf{e}_{1}=0, \quad \mathbf{x} \in S_{i}^{ \pm}, i=f, s, 0<t<T
$$

by introducing a new pressure

$$
p_{f} \rightarrow p_{f}-p^{0}(\mathbf{x}), \quad p_{s} \rightarrow p_{s}-p^{0}(\mathbf{x}), \quad p^{0}(\mathbf{x})=\frac{1}{2} p^{+}(\mathbf{x})\left(1-x_{1}\right)
$$

With this new pressure the dynamic equations take the form

$$
\begin{aligned}
& \nabla \cdot \mathbb{P}_{f}+\mathbf{f}+\rho_{f} \mathbf{e}=0, \quad \nabla \cdot \mathbf{u}^{f}=0, \quad \mathbf{x} \in Q_{f}, 0<t<T ; \\
& \nabla \cdot \mathbb{P}_{s}+\mathbf{f}=0, \quad \nabla \cdot \mathbf{u}^{s}=0, \quad \mathbf{x} \in Q_{s}, 0<t<T,
\end{aligned}
$$

where

$$
\mathbf{f}(\mathbf{x})=(1-\chi(\mathbf{x})) \rho_{s} \mathbf{e}+\nabla p^{0}(\mathbf{x})
$$

and

$$
\chi(\mathbf{x})=1, \quad \text { for } \mathbf{x} \in Q_{f}, \quad \text { and } \quad \chi(\mathbf{x})=0, \quad \text { for } \mathbf{x} \in Q_{s} .
$$

Finally

$$
\mathbf{u}^{s}(\mathbf{x}, 0)=0, \quad \mathbf{x} \in S .
$$


The initial and boundary conditions for the density are equivalent to specifying the surface $\Gamma_{0}$ that separates two subdomains $Q_{f}^{ \pm}(0)$ initially occupied by different fluids. For the sake of simplicity we suppose that

$$
\Gamma^{(0)}=\left\{\mathbf{x} \in Q_{f}: x_{1}=h\left(x_{2}\right),-\frac{1}{2}<x_{2}<\frac{1}{2}\right\}
$$

and

$$
-\frac{1}{2}+\delta<h\left(x_{2}\right)<\frac{1}{2}-\delta, \quad \text { for }-\frac{1}{2}<x_{2}<\frac{1}{2}
$$

with some $0<\delta<1$.

So, we may expect that the free boundary $\Gamma(t)$ will not touch the given boundaries $S^{ \pm}$ at least for some time interval $0<t<T$.

At the boundaries $S^{ \pm}$for $0<t<T$ and at initial moment $t=0$, the density $\rho_{f}$ is piecewise constant and assumes two positive values characterizing the distinct phases of the flow

$$
\begin{aligned}
& \rho_{f}(\mathbf{x}, t)=\rho^{ \pm}=\text {const }>0, \quad \mathbf{x} \in S_{f}^{ \pm}, 0<t<T, \\
& \rho_{f}(\mathbf{x}, 0)=\rho_{0}(\mathbf{x}), \quad \mathbf{x} \in Q_{f},
\end{aligned}
$$

where $\rho_{0}(\mathbf{x})=\rho^{ \pm}$for $\mathbf{x} \in Q_{f}^{ \pm}(0)$.

Suppose for simplicity that

$$
\rho_{0}^{-} \leq \rho_{0}(\mathbf{x}) \leq \rho_{0}^{+}
$$

If the velocity $\mathbf{u}^{f}(\mathbf{x}, t)$ of the liquid is sufficiently smooth, then the Cauchy problem

$$
\frac{d \mathbf{x}}{d t}=\mathbf{u}^{f}(\mathbf{x}, t), \quad t>t_{0},\left.\mathbf{x}\right|_{t=t_{0}}=\xi
$$

determines a mapping

$$
\mathbf{x}=\gamma\left(\xi, t ; \mathbf{u}^{f} ; t_{0}\right), \quad \gamma: Q_{f} \rightarrow Q_{f}
$$

In particular, the free boundary $\Gamma(t)$ is determined as a set

$$
\Gamma(t)=\left\{\mathbf{x} \in Q_{f}: \mathbf{x}=\gamma\left(\xi, t ; \mathbf{u}^{f} ; 0\right), \xi \in \Gamma(0)\right\},
$$

and subdomains $Q_{f}^{ \pm}(t)=\left\{\mathbf{x} \in Q_{f}: \rho_{f}(\mathbf{x}, t)=\rho^{ \pm}\right\}$as sets

$$
\begin{aligned}
Q_{f}^{ \pm}(t)= & \left\{\mathbf{x} \in Q_{f}: \mathbf{x}=\boldsymbol{\gamma}\left(\boldsymbol{\xi}, t ; \mathbf{u}^{f} ; 0\right), \xi \in Q_{f}^{ \pm}(0)\right\} \\
& \cap\left\{\mathbf{x} \in Q_{f}: \mathbf{x}=\boldsymbol{\gamma}\left(\xi, t ; \mathbf{u}^{f} ; t_{0}\right), \boldsymbol{\xi} \in S_{f}^{ \pm}, t_{0}>0\right\} .
\end{aligned}
$$

The problem treated here is that of finding the velocity $\mathbf{u}^{f}(\mathbf{x}, t)$ and pressure $p_{f}(\mathbf{x}, t)$ of the liquid in pores, the displacement $\mathbf{u}^{s}(\mathbf{x}, t)$ and pressure $p_{s}(\mathbf{x}, t)$ of the solid skeleton, and the density $\rho_{f}(\mathbf{x}, t)$ of the liquid from the above equations and the initial and boundary data. Note that it is nonlinear because of the coupling term $\mathbf{u}^{f} \cdot \nabla \rho_{f}$ in (2). 
It is shown below that the evolution described by the above equations preserves the existence of two subdomains $Q_{f}^{ \pm}(t)$, each occupied by one of the fluids, that are separated at time $t>0$ by a regular free boundary $\Gamma(t)$. Thus, the problem studied is equivalent to finding $\left\{\mathbf{u}^{f}, p_{f}, \mathbf{u}^{s}, p_{s}\right\}$, and the moving boundary $\Gamma(t)$.

Theorems on the existence of generalized solutions to the Navier-Stokes system for nonhomogeneous incompressible fluids were obtained in, e.g., [2-9] (without a detailed analysis of the set where the density is discontinuous). The existence and uniqueness of the classical solution to the Stokes equations for a non-homogeneous liquid with Dirichlet data have been proved in [10], and with Neumann data in [1]. The Muskat problem at the microscopic level with corresponding homogenization has been considered in [11].

Finally, we explain our motivation to study this particular problem. It is well known [12] that the Darcy system of filtration, describing the macroscopic flow of a homogeneous incompressible liquid in some bounded domain $Q$, is a result of homogenization of the Stokes system for an incompressible viscous liquid occupying a periodic pore space $Q_{\varepsilon} \subset$ $Q$ in an absolutely rigid solid body.

The more complicated macroscopic motion of two immiscible incompressible viscous liquids is governed by the Muskat problem. In this model one looks for the free boundary $\Gamma(t) \subset Q$, which separates two different domains $Q^{+}(t) \subset Q$ and $Q^{-}(t) \subset Q, Q^{+}(t) \cup \Gamma(t) \cup$ $Q^{-}(t)=Q$, occupied by different fluids. In each of the domains $Q^{ \pm}(t)$ the liquid motion is described by its own Darcy system of filtration, and at the free boundary the normal velocities of the liquids coincide with the normal velocity of the free boundary.

Thus, we may expect that, as in the case of the filtration of a single liquid, the Muskat problem should be a homogenization of the initial boundary value problem for the Stokes system with a non-homogeneous liquid,

$$
\mu \triangle \mathbf{u}^{\varepsilon}+g \rho_{\varepsilon} \mathbf{e}=0, \quad \nabla \cdot \mathbf{u}^{\varepsilon}=0, \quad \frac{d \rho_{\varepsilon}}{d t}=0,
$$

in a periodic pore space $Q_{\varepsilon}$ of an absolutely rigid solid body $Q$ with the following boundary and initial conditions:

$$
\begin{aligned}
& \mathbf{u}^{\varepsilon}(\mathbf{x}, t)=0, \quad \mathbf{x} \in \partial Q_{\varepsilon}, \\
& \rho_{\varepsilon}(\mathbf{x}, 0)=\rho_{\varepsilon}^{0}(\mathbf{x}), \quad \mathbf{x} \in Q_{\varepsilon},
\end{aligned}
$$

where

$$
\rho_{\varepsilon}^{0}(\mathbf{x})=\rho^{+}=\text {const }, \quad \mathbf{x} \in Q_{\varepsilon}^{+}(0), \quad \rho_{\varepsilon}^{0}(\mathbf{x})=\rho^{-}=\text {const }, \quad \mathbf{x} \in Q_{\varepsilon}^{-}(0),
$$

$\overline{Q_{\varepsilon}^{+}}(0) \cup \overline{Q_{\varepsilon}^{-}}(0)=\overline{Q_{\varepsilon}}, \mu$ is the viscosity and $g \mathbf{e}$ is the acceleration due to gravity.

But until now this fact has not been proven and it may not be true for an absolutely rigid solid body.

Some indirect arguments confirm this guess.

As a first argument, note that the problem (17)-(19) possesses the evident a priori estimate

$$
\max _{0<t<T} \mu^{2} \int_{Q_{\varepsilon}}\left|\nabla \mathbf{u}^{\varepsilon}(\mathbf{x}, t)\right|^{2} d x<C_{0},
$$

where $C_{0}$ is independent of $\varepsilon$. 
In order to pass to the limit in the transport equation (2) for the density $\rho_{\varepsilon}$, as the size $\varepsilon$ of pores goes to zero, one needs at least uniform boundedness of the gradient of the velocity $\mathbf{u}^{\varepsilon}$ :

$$
\int_{Q_{\varepsilon}}\left|\nabla \mathbf{u}^{\varepsilon}(\mathbf{x}, t)\right|^{2} d x<C_{1}
$$

with constant $C_{1}$ independent of $\varepsilon$.

On the other hand, the Friedrichs-Poincaré inequality in the periodic cell of an absolutely rigid skeleton ([12], equation (1.1.8), p.4) for the velocity,

$$
\int_{Q_{\varepsilon}} \mu\left|\nabla \mathbf{u}^{\varepsilon}(\mathbf{x}, t)\right|^{2} d x+\int_{Q_{\varepsilon}}\left|\mathbf{u}^{\varepsilon}(\mathbf{x}, t)\right|^{2} d x \leq C_{2} \frac{\varepsilon^{2}}{\mu},
$$

dictates the unique asymptotic behavior of the viscosity $\mu$ as $\varepsilon \rightarrow 0$ :

$$
\mu=\mu_{1} \varepsilon^{2}
$$

where $\mu_{1}$ and $C_{2}$, like the constant $C_{1}$ in the previous estimate, are independent of $\varepsilon$.

Thus, condition (23) and estimate (20) do not guarantee estimate (21), and, consequently, the compactness of the sequence $\left\{\mathbf{u}^{\varepsilon}\right\}$. Without this compactness we cannot pass to the limit in the transport equation for the density $\rho^{\varepsilon}$ and get the desired result.

The second argument comes from the problem (17)-(19). For simplicity we consider a system of disjoint cylindric capillaries, with parallel axes and the motion of liquids under constant pressures at the 'entrance' $S^{+}$and 'exit' $S^{-}$boundaries.

Due to the boundary condition (18), the contact points of the free boundary and the solid skeleton will be permanently fixed at the initial position. Numerical implementations predict the appearance of a water tongue, which grows with time (see Figure 1). The

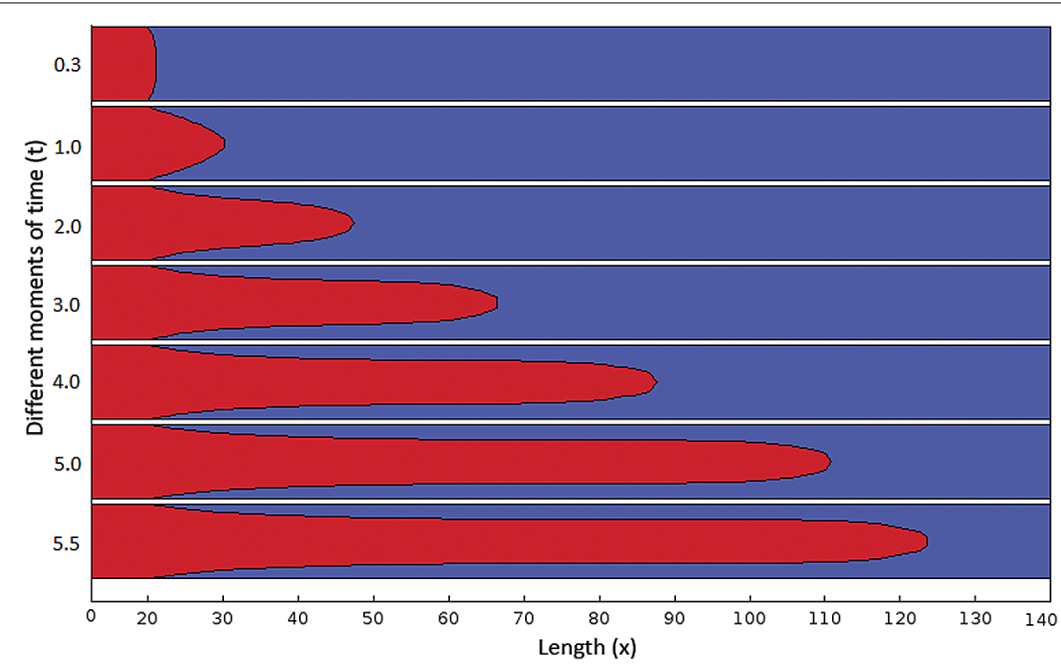

Figure 1 Numerical simulation: successive positions of the free boundary in a single capillary. Due to the boundary condition (18), the contact points of the free boundary and the solid skeleton will be permanently fixed at the initial position. Numerical implementations predict the appearance of a water tongue, which grows with time. 


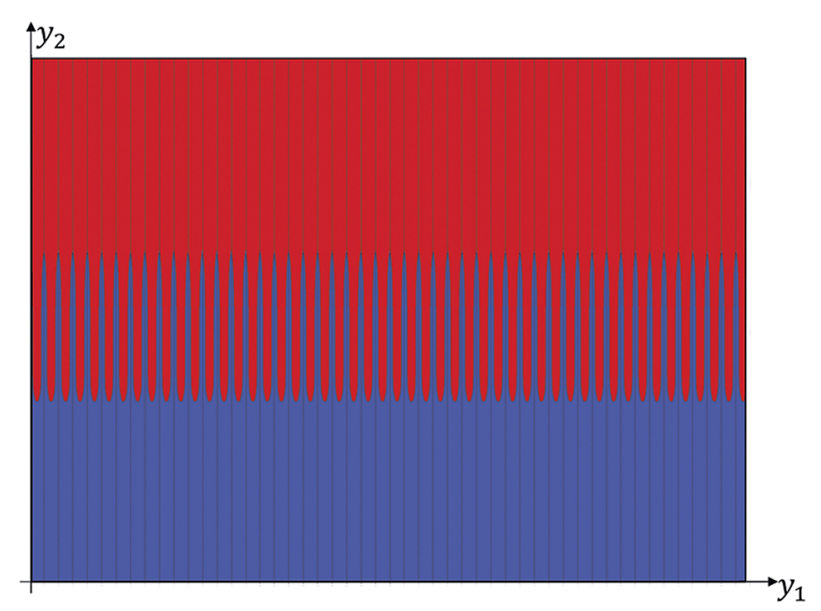

Figure 2 Numerical homogenization for $\boldsymbol{t}=\mathbf{5 . 5}$. The gradual growth of the number of capillaries leads to homogenization of the liquid motion.

Figure 3 Homogenization by increasing the number of capillaries. Concentration of water $s$ for increasing times (left to right). The domain occupied by the water tongues at a fixed time becomes under homogenization a mushy region, where the concentration $s$ of water varies from 1 to 0 .

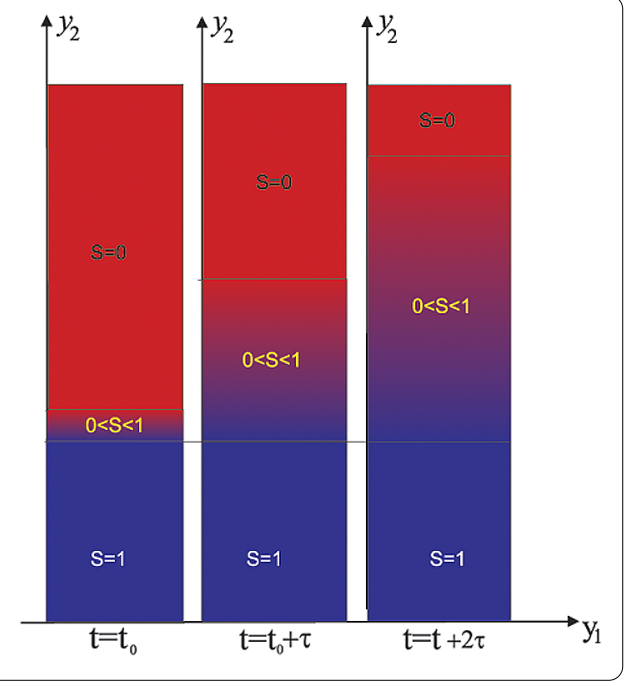

gradual growth of the number of capillaries (Figure 2) leads to homogenization of the liquid motion. The domain occupied by the water tongues at a fixed time becomes under homogenization a mushy region, where the concentration $s$ of water varies from 1 to 0 (Figures 3 and 4).

Now, if we return to the Muskat problem, we may see that the solution of the Muskat problem corresponding to the macroscopic joint motion of two different liquids has a very simple structure. The free boundary separates two liquids and moves with a constant velocity (Figure 5).

So, we cannot obtain the Muskat problem of the liquid motion in the pore space of an absolutely rigid body as a homogenization of the corresponding initial boundary value problem for a Stokes system with a non-homogeneous liquid.

But if we look for the motion of a non-homogeneous liquid in an elastic solid body (the problem (2)-(6), (8), (10) (13), (14)), then the situation changes. The contact points of the 


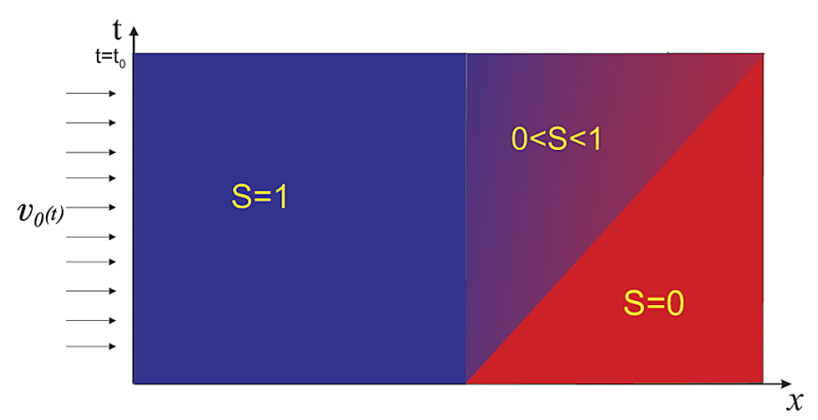

Figure 4 The limit of rigorous numerical upscaling. The domain occupied by the water tongues at a fixed time becomes under homogenization a mushy region, where the concentration $s$ of water varies from 1 to 0 .

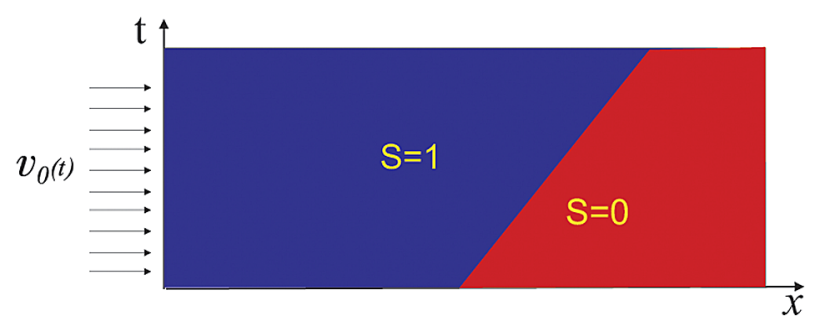

Figure 5 The solution of the Muskat problem. The free boundary separates two liquids and moves with a constant velocity.

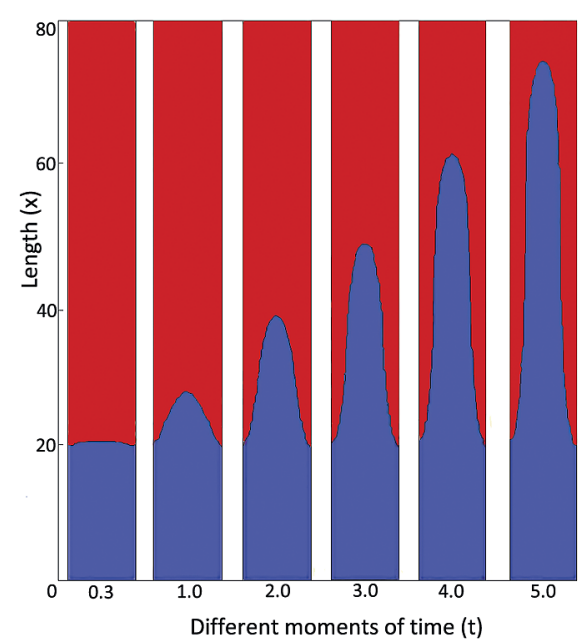

(a)

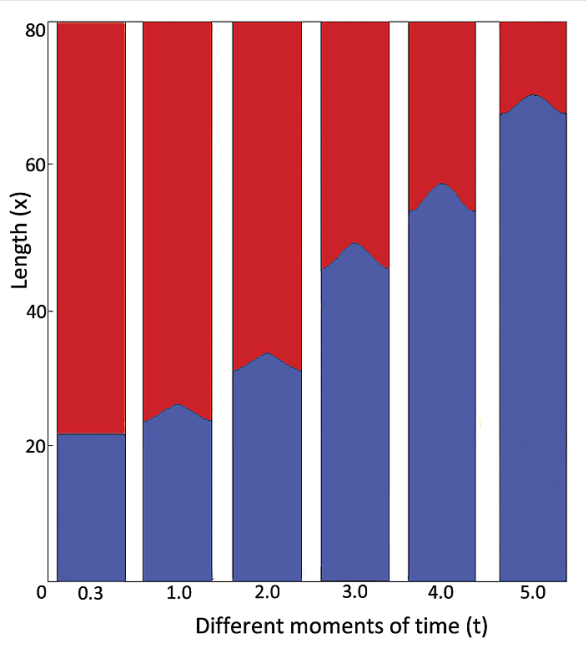

(b)

Figure 6 Displacement of oil $\left(\rho^{+}=1.1\right)$ by water $\left(\rho^{-}=0.86\right)$. (a) In an absolutely rigid solid and (b) in an elastic solid skeleton with the elasticity coefficient $\lambda=15$. The contact points of the free boundary and solid body begin to move, and homogenization conserves the free boundary which separates the two liquids.

free boundary and solid body begin to move (Figure 6), and homogenization conserves the free boundary which separates the two liquids.

The aim of this paper is to show that the problem (2)-(6), (8), (10) (13) and (14) for an elastic solid body has a solution with a smooth free boundary, which divides the two liquids. 


\section{The main result}

Throughout the article, we use the customary notation of function spaces and norms (see, e.g., [13], pp.4-7). Thus, for $1<q<\infty$

$$
\begin{aligned}
& u \in L_{q}(\Omega) \quad \Rightarrow \quad\|u\|_{q, \Omega}=\left(\int_{\Omega}|u|^{q} d x\right)^{\frac{1}{q}}<\infty, \\
& u \in L_{\infty}(\Omega) \quad \Rightarrow \quad\|u\|_{\infty, \Omega}=\lim _{q \rightarrow \infty}\|u\|_{q, \Omega}<\infty, \\
& u \in W_{q}^{1}(\Omega) \quad \Rightarrow \quad\|u\|_{q, \Omega}^{(1)}=\left(\int_{\Omega}|u|^{q} d x\right)^{\frac{1}{q}}+\sum_{i=1}^{2}\left(\int_{\Omega}\left|\frac{\partial u}{\partial x_{i}}\right|^{q} d x\right)^{\frac{1}{q}}<\infty, \\
& u \in \stackrel{\circ}{q}_{q}^{1}(\Omega) \quad \Rightarrow \quad u \in W_{q}^{1}(\Omega), \quad \text { and } \quad u(\mathbf{x})=0, \quad \mathbf{x} \in \partial \Omega, \\
& u \in W_{q}^{l}(\Omega) \quad \Rightarrow \quad\|u\|_{q, \Omega}^{(l)}=\left(\int_{\Omega}|u|^{q} d x\right)^{\frac{1}{q}}+\sum_{|m|=l}\left(\int_{\Omega}\left|D^{m} u\right|^{q} d x\right)^{\frac{1}{q}}<\infty, \\
& D^{m} u=\frac{\partial^{|m|} u}{\partial^{m_{1}} x_{1} \cdots \partial^{m_{n} x_{n}}}, \quad m=\left(m_{1}, \ldots, m_{n}\right), m_{i} \geq 0,|m|=m_{1}+\cdots+m_{n} .
\end{aligned}
$$

Next we introduce the space of functions with non-integer derivatives. To do this straightforwardly we consider the half-spaces

$$
\begin{aligned}
& \mathbb{R}_{f}^{2}=\left\{\mathbf{x}=\left(x_{1}, x_{2}\right) \in \mathbb{R}^{2}:\left|x_{1}\right|<\infty, x_{2}>\frac{1}{2}\right\}, \\
& \mathbb{R}_{s}^{2}=\left\{\mathbf{x}=\left(x_{1}, x_{2}\right) \in \mathbb{R}^{2}:\left|x_{1}\right|<\infty, x_{2}<\frac{1}{2}\right\},
\end{aligned}
$$

with the boundary

$$
\mathbb{R}=\left\{\mathbf{x} \in \mathbb{R}^{2}:\left|x_{1}\right|<\infty, x_{2}=\frac{1}{2}\right\}
$$

The space $W_{2}^{l-\frac{1}{2}}(\mathbb{R})$ is the space of all functions $v\left(x_{1}\right)$ with a finite norm

$$
\|v\|_{2, \mathbb{R}}^{\left(l-\frac{1}{2}\right)}=\left(\int_{-\infty}^{\infty}|\xi|^{2 l-1}|\widehat{v}(\xi)|^{2} d \xi\right)^{\frac{1}{2}}
$$

where $\widehat{v}$ is a Fourier transformation of $v$ :

$$
\widehat{v}(\xi)=\frac{1}{\sqrt{2 \pi}} \int_{-\infty}^{\infty} v\left(x_{1}\right) e^{-i \xi x_{1}} d x_{1} .
$$

According to [13] (Chapter 2, Theorem 2.3, p.71)

$$
\|v\|_{2, \mathbb{R}}^{\left(l-\frac{1}{2}\right)} \leq C_{1}\|v\|_{2, \mathbb{R}_{j}^{2}}^{(l)} \leq C_{2}\|v\|_{2, \mathbb{R}}^{\left(l-\frac{1}{2}\right)}, \quad j=f, s .
$$

For smooth functions we define the following norms:

$$
|u|_{\Omega}^{(0)}=\sup _{\mathbf{x} \in \Omega}|u(\mathbf{x})|, \quad\langle u\rangle_{\Omega}^{(\alpha)}=\sup _{\mathbf{x} \in \Omega} \frac{|u(\mathbf{x})-u(\mathbf{y})|}{|x-\mathbf{y}|^{\alpha}} .
$$


We say that the function $u(\mathbf{x})$ belongs to the space $C^{\alpha}(\bar{\Omega})$ if

$$
|u|_{\Omega}^{(\alpha)}=|u|_{\Omega}^{(0)}+\langle u\rangle_{\Omega}^{(\alpha)}<\infty
$$

it belongs to the space $C^{k}(\bar{\Omega})$ if

$$
|u|_{\Omega}^{(k)}=\sum_{|m|=0}^{k}\left|D^{m} u\right|_{\Omega}^{(0)}<\infty
$$

and it belongs to the space $C^{k+\alpha}(\bar{\Omega})$ if

$$
|u|_{\Omega}^{(k+\alpha)}=|u|_{\Omega}^{(k)}+\sum_{|m|=0}^{k}\left|D^{m} u\right|_{\Omega}^{(\alpha)}<\infty .
$$

We say that the surface $\Gamma \in \Omega$ is $C^{k+\alpha}$ regular if in local coordinates it is presented by $C^{k+\alpha}$ regular functions.

If $u=u(\mathbf{x}, t)$ and $u(\mathbf{x}, t) \in \mathbb{B}$ for all $0<t<T$, then

$$
u \in L_{q}((0, T) ; \mathbb{B}) \Longleftrightarrow \int_{0}^{T}\|\mathbf{u}(\cdot, t)\|_{B}^{q} d t<\infty,
$$

and for $q=\infty$

$$
u \in L_{\infty}((0, T) ; \mathbb{B}) \Longleftrightarrow \sup _{0<t<T}\|\mathbf{u}(\cdot, t)\|_{B}<\infty
$$

Finally, $u \in C^{2,1}\left(\overline{\Omega_{T}}\right), \Omega_{T}=\Omega \times(0, T)$, if

$$
\max _{0<t<T}\left(|\mathbf{u}(\cdot, t)|_{\Omega}^{(2)}+\left|\frac{\partial u}{\partial t}(\cdot, t)\right|_{\Omega}^{(0)}\right)<\infty
$$

For any $0<\delta<1$ we put

$$
\begin{aligned}
& Q^{(\delta)}=\left\{\mathbf{x} \in Q:-1+\delta<x_{1}<1-\delta\right\}, \quad Q_{f}^{(\delta)}=Q^{(\delta)} \cap Q_{f}, \\
& G^{(\delta)}=Q^{(\delta)} \times(0, T), \quad G_{f}=Q_{f} \times(0, T), \quad G_{f}^{(\delta)}=Q_{f}^{(\delta)} \times(0, T) .
\end{aligned}
$$

Our principal result is the following.

Theorem 2.1 Under the condition

$$
\begin{aligned}
& \|\mathbf{f}\|_{\infty, Q}=C^{0}<\infty, \\
& \Gamma(0) \in C^{1+\alpha}, \quad 0<\alpha<1,
\end{aligned}
$$

the problem (2)-(4), (6), (8), (10) (13), (14) has a unique solution in the interval [0,T) for some $T>0$.

The elements of this solution possess the following properties. 
(i) For any $0<\delta<1$, and $0<\alpha<1$, the velocity $\mathbf{u}$ and pressure $p$ satisfy the regularity conditions

$$
\mathbf{u} \in L_{\infty}\left(0, T ; W_{2}^{3}\left(Q^{(\delta)}\right)\right) \cap L_{\infty}\left(0, T ; C^{1+\alpha}\left(Q^{(\delta)}\right)\right), \quad p \in L_{\infty}\left(0, T ; W_{2}^{2}\left(Q^{(\delta)}\right)\right),
$$

equations (8) almost everywhere in $Q \times(0, T)$, boundary conditions (4), (13), and initial conditions (10) and (14) in the usual sense, and boundary conditions (3) and (6) in the sense of distributions as an integral identity

$$
\int_{\Omega}(\mathbb{P}(\mathbf{u}(t), p(t)): \mathbb{D}(\boldsymbol{\varphi})+\mathbf{f} \cdot \boldsymbol{\varphi}) d x=0
$$

for almost all $0<t<T$ and for any smooth solenoidal functions $\boldsymbol{\varphi}$ vanishing at $\mathbf{x} \in S^{0}$.

(ii) The free boundary $\Gamma(t)$ is a surface of class $C^{1, \alpha}$ at each time $t \in[0, T)$, and the normal velocity $V_{n}(\mathbf{x}, t)$ of the free boundary in the direction of its normal $\mathbf{n}$ at position $\mathbf{x}$ is uniformly bounded,

$$
\sup _{\substack{t \in(0, T) \\ \mathbf{x} \in \Gamma(t)}}\left|V_{n}(\mathbf{x}, t)\right|<\infty
$$

(iii) The density $\rho$ has bounded variation,

$$
\rho \in L_{\infty}\left(0, T ; B V\left(Q^{(\delta)}\right)\right) \cap B V\left(Q^{(\delta)} \times(0, T)\right)
$$

and it satisfies the transport equation (2) in the sense of distributions

$$
\int_{\Omega_{T}} \rho\left(\frac{\partial \psi}{\partial t}+u \cdot \nabla \psi\right) d x d t=-\int_{\Omega} \rho_{0}(\mathbf{x}) \psi(\mathbf{x}, 0) d x
$$

for any smooth functions $\psi$, vanishing at $t=T$ and $\mathbf{x} \in S^{ \pm}$.

The time $T$ of the existence of the classical solution depends on the behavior of the free boundary $\Gamma(t)$. Namely, let $\delta^{ \pm}(t)$ be the distance between $\Gamma(t)$ and the boundary $S^{ \pm}$and $\delta(t)=\min \left(\delta^{-}(t), \delta^{+}(t)\right)$. Then $\delta(t)>0$ for all $0<t<T$ and $\delta(t) \rightarrow 0$ as $t \rightarrow T$.

\section{Proof of the main result}

Let

$$
\begin{aligned}
& \rho_{0}^{\varepsilon}(\mathbf{x})=\mathbf{M}_{\varepsilon}^{(2)}\left(\rho_{0}\right)=\frac{1}{\varepsilon^{2}} \int_{Q_{f}} J\left(\frac{|\mathbf{x}-\mathbf{y}|}{\varepsilon}\right) \rho_{0}(\mathbf{y}) d y, \quad \mathbf{x} \in Q_{f}, \\
& \rho_{0}^{\varepsilon} \in C^{\infty}\left(Q_{f}\right), \quad \rho_{0}^{\varepsilon}(\mathbf{x}) \rightarrow \rho_{0}(\mathbf{x}) \quad \text { a.e. in } Q_{f}^{ \pm}(0),
\end{aligned}
$$

where

$$
\begin{aligned}
& J(s) \geq 0, \quad J(s)=0 \quad \text { for }|s|>1, \\
& J(s)=J(-s), \quad J \in C^{\infty}(-\infty,+\infty), \quad \int_{\mathbb{R}^{2}} J(|x|) d x=1 .
\end{aligned}
$$

We divide the proof of Theorem 2.1 into several steps. 
3.1 We show that for each $\varepsilon>0$ the modified problem

$$
\begin{aligned}
& \nabla \cdot \mathbb{P}_{f}^{\varepsilon}+\rho^{\varepsilon} \mathbf{e}+\mathbf{f}=0, \quad \nabla \cdot \mathbf{u}^{f, \varepsilon}=0, \quad \mathbf{x} \in Q_{f}, 0<t<T, \\
& \nabla \cdot \mathbb{P}_{s}^{\varepsilon}+\mathbf{f}=0, \quad \nabla \cdot \mathbf{u}^{s, \varepsilon}=0, \quad \mathbf{x} \in Q_{s}, 0<t<T, \\
& \mathbf{u}^{s, \varepsilon}(\mathbf{x}, 0)=0, \quad \mathbf{x} \in S, \\
& \mathbf{u}^{f, \varepsilon}=\frac{\partial \mathbf{u}^{s, \varepsilon}}{\partial t}, \quad \mathbb{P}_{f}^{\varepsilon} \cdot \mathbf{n}=\mathbb{P}_{s}^{\varepsilon} \cdot \mathbf{n}, \quad \mathbf{x} \in S, 0<t<T, \\
& \mathbb{P}_{i}^{\varepsilon} \cdot \mathbf{e}_{1}=0, \quad \mathbf{x} \in S_{i}^{ \pm}, i=f, s, \quad \mathbf{u}^{s, \varepsilon}(\mathbf{x}, t)=0, \quad \mathbf{x} \in S^{0}, 0<t<T, \\
& \mathbb{P}_{f}\left(\mathbf{u}^{f, \varepsilon}, p_{f}^{\varepsilon}\right)=2 \mu \mathbb{D}\left(\mathbf{u}^{f, \varepsilon}\right)-p_{f}^{\varepsilon} \mathbb{I}, \quad \mathbb{P}_{s}\left(\mathbf{u}^{s, \varepsilon}, p_{s}^{\varepsilon}\right)=2 \lambda \mathbb{D}\left(\mathbf{u}^{s, \varepsilon}\right)-p_{s}^{\varepsilon} \mathbb{I}, \\
& \frac{\partial \rho^{\varepsilon}}{\partial t}+\mathbf{v}^{\varepsilon} \cdot \nabla \rho^{\varepsilon}=0, \quad \mathbf{x} \in Q, 0<t<T, \\
& \rho^{\varepsilon}(\mathbf{x}, t)=\rho_{0}^{ \pm}, \quad \mathbf{x} \in S_{f}^{ \pm}, 0<t<T, \\
& \rho^{\varepsilon}(\mathbf{x}, 0)=\rho_{0}^{\varepsilon}(\mathbf{x}), \quad \mathbf{x} \in Q, \quad \mathbf{v}^{\varepsilon}=\mathbf{M}_{\varepsilon}^{(1)}\left(\mathbf{M}_{\varepsilon}^{(2)}\left(\mathbf{u}^{f, \varepsilon}\right)\right), \\
& \mathbf{M}_{\varepsilon}^{(1)}(\mathbf{v})=\frac{1}{\varepsilon} \int_{0}^{\infty} J\left(\frac{|t-\tau|}{\varepsilon}\right) \mathbf{v}(\mathbf{x}, \tau) d \tau,
\end{aligned}
$$

of finding $\mathbf{u}^{f, \varepsilon}, p_{f}^{\varepsilon}, \rho^{\varepsilon}, \mathbf{u}^{s, \varepsilon}, p_{s}^{\varepsilon}$ has at least one classical solution.

To solve the problem (29), (30) we use the Schauder fixed point theorem [14].

Namely, let $\mathcal{M}$ be the set of all continuous functions

$$
\tilde{\rho} \in C(\bar{G}), \quad G=Q_{f} \times(0, T),
$$

such that

$$
\rho^{-} \leq \widetilde{\rho}(\mathbf{x}, t) \leq \rho^{+}
$$

For fixed $\varepsilon>0$ we define the following nonlinear operator:

$$
\Phi: \mathcal{M} \rightarrow \mathcal{M}, \quad \rho=\Phi(\widetilde{\rho})
$$

and

3.1.1 prove that the linear problem

$$
\begin{aligned}
& \nabla \cdot \mathbb{P}_{f}+\tilde{\rho}^{\varepsilon} \mathbf{e}+\mathbf{f}=0, \quad \nabla \cdot \mathbf{u}^{f}=0, \quad \mathbf{x} \in Q_{f}, 0<t<T, \\
& \nabla \cdot \mathbb{P}_{s}+\mathbf{f}=0, \quad \nabla \cdot \mathbf{u}^{s}=0, \quad \mathbf{x} \in Q_{s}, 0<t<T, \\
& \mathbf{u}^{s}(\mathbf{x}, 0)=0, \quad \mathbf{x} \in S, \\
& \mathbf{u}^{f}=\frac{\partial \mathbf{u}^{s}}{\partial t}, \quad \mathbb{P}_{f} \cdot \mathbf{n}=\mathbb{P}_{s} \cdot \mathbf{n}, \quad \mathbf{x} \in S, 0<t<T, \\
& \mathbb{P}_{i} \cdot \mathbf{e}_{1}=0, \quad \mathbf{x} \in S_{i}^{ \pm}, i=f, s, \quad \mathbf{u}^{s}(\mathbf{x}, t)=0, \quad \mathbf{x} \in S^{0}, 0<t<T, \\
& \frac{\partial \rho}{\partial t}+\mathbf{v}^{\varepsilon} \cdot \nabla \rho=0, \quad \mathbf{x} \in Q, 0<t<T, \\
& \rho(\mathbf{x}, t)=\rho_{0}^{ \pm}, \quad \mathbf{x} \in S_{f}^{ \pm}, 0<t<T, \quad \rho(\mathbf{x}, 0)=\rho_{0}^{\varepsilon}(\mathbf{x}), \quad \mathbf{x} \in Q,
\end{aligned}
$$




$$
\begin{aligned}
& \widetilde{\rho}^{\varepsilon}(\mathbf{x}, t)=\mathbf{M}_{\varepsilon}^{(1)}(\widetilde{\rho}), \quad \mathbf{v}^{\varepsilon}=\mathbf{M}_{\varepsilon}^{(1)}\left(\mathbf{M}_{\varepsilon}^{(2)}\left(u^{f}\right)\right), \\
& \mathbb{P}_{f}=2 \mu \mathbb{D}\left(\mathbf{u}^{f}\right)-p_{f} \mathbb{I}, \quad \mathbb{P}_{s}=2 \lambda \mathbb{D}\left(\mathbf{u}^{s}\right)-p_{s} \mathbb{I},
\end{aligned}
$$

for given $\widetilde{\rho} \in \mathcal{M}$ has a unique weak solution $\left\{\mathbf{u}^{f}, p_{f}, \mathbf{u}^{s}, p_{s}, \rho=\Phi(\widetilde{\rho})\right\}$.

The properties of the mollifier $\mathbf{M}_{\varepsilon}^{(2)}$ and continuity equation for $u$ imply the continuity equation for $\mathbf{v}^{\varepsilon}$ :

$$
\nabla \cdot \mathbf{v}^{\varepsilon}=0, \quad \mathbf{x} \in Q_{f}, 0<t<T .
$$

3.1.2 To prove the solvability of (29), (30) we show that the operator $\Phi$ is completely continuous and according to Schauder's fixed point theorem it has at least one fixed point in $\mathcal{M}$.

3.1.3 Finally, we prove that the modified problem (29), (30) has a unique solution.

3.2 In this part of the proof we derive uniform bounds for the solutions of the modified problem (29), (30).

More precisely, we first derive

3.2.1 L $L_{2}$-estimates for the solutions of the modified problem (29), (30),

and using the Fourier transform's techniques we find

3.2.2 uniform estimates for the solutions of the problem (29), (30) in Hölder's spaces.

This part of the proof contains a lot of technical details and is very difficult to follow. Unfortunately, the linear problem that arises is completely novel and requires special consideration. The standard method for classical differential equations consists of:

(1) a Fourier transform of the original problem,

(2) exact representation of the solution of the corresponding linear problem in new variables, and

(3) inverse transformation and derivation of the exact representation of the solution in the original variables.

In our case the linear problem in new variables for the Fourier transform of the solution is still complicated and has no exact representation. This is why we estimate only a Fourier transform of the solution and after that use Parseval's equality to get estimates for the solution in the original variables.

3.3 Finally we derive uniform estimates for the densities and prove the existence of a smooth surface separating the parts of the domain occupied by the two different fluids.

In what follows, by $C$ we denote constants depending only on $C^{0}, \rho^{ \pm}$, and $\rho_{s}$.

\subsection{Solvability of the modified problem (29), (30)}

Definition 1 We say that a set of functions $\left\{\mathbf{u}^{f, \varepsilon}, p_{f}^{\varepsilon}, \mathbf{u}^{s, \varepsilon}, p_{s}^{\varepsilon}, \rho^{\varepsilon}\right\}$

$$
\begin{aligned}
& \mathbf{u}^{i, \varepsilon} \in L_{\infty}\left((0, T) ; W_{2}^{1}\left(Q_{i}\right)\right), \quad p_{i}^{\varepsilon} \in L_{\infty}\left((0, T) ; L_{2}\left(Q_{i}\right)\right), \quad i=f, s, \\
& \rho^{\varepsilon} \in C^{1}\left(\overline{Q_{T}}\right), \quad Q_{T}=Q \times(0, T),
\end{aligned}
$$


is a weak solution of the problem (29), (30), if it satisfies the integral identity

$$
\begin{aligned}
\int_{Q_{f}} & \mathbb{P}_{f}\left(\mathbf{u}^{f, \varepsilon}, p_{f}^{\varepsilon}\right): \mathbb{D}(\boldsymbol{\varphi}) d x+\int_{Q_{s}} \mathbb{P}_{s}\left(\mathbf{u}^{s, \varepsilon}, p_{s}^{\varepsilon}\right): \mathbb{D}(\boldsymbol{\varphi}) d x \\
= & \int_{Q_{f}} \rho^{\varepsilon}(\mathbf{e} \cdot \boldsymbol{\varphi}) d x+\int_{Q} \mathbf{f} \cdot \boldsymbol{\varphi} d x,
\end{aligned}
$$

for almost all $t \in(0, T)$, and for arbitrary smooth functions $\varphi(\mathbf{x})$, vanishing at $S^{0}$, and the problem (30) in the usual sense.

\subsubsection{The solvability of the problem (33) for given $\tilde{\rho}$}

Lemma 1 Under the same conditions of Theorem 2.1 the problem (33) for given $\widetilde{\rho}$ has a unique weak solution,

$$
\mathbf{u}^{i} \in L_{\infty}\left((0, T) ; W_{2}^{1}\left(Q_{i}\right)\right), \quad i=f, s, \quad \frac{\partial \mathbf{u}^{s}}{\partial t} \in L_{2}\left((0, T) ; W_{2}^{1}\left(Q_{s}\right)\right),
$$

satisfying the following estimates:

$$
\begin{aligned}
& \int_{0}^{T} \int_{Q_{f}} \mathbb{D}\left(\mathbf{u}^{f}\right): \mathbb{D}\left(\mathbf{u}^{f}\right) d x d t+\max _{0<t<T} \int_{Q_{s}} \mathbb{D}\left(\mathbf{u}^{s}(\mathbf{x}, t)\right): \mathbb{D}\left(\mathbf{u}^{s}(\mathbf{x}, t)\right) d x \leq C, \\
& \max _{0<t<T} \int_{Q_{f}} \mathbb{D}\left(\mathbf{u}^{f}(\mathbf{x}, t)\right): \mathbb{D}\left(\mathbf{u}^{f}(\mathbf{x}, t)\right) d x \\
& \quad+\int_{0}^{T} \int_{Q_{s}} \mathbb{D}\left(\frac{\partial \mathbf{u}^{s}}{\partial t}\right): \mathbb{D}\left(\frac{\partial \mathbf{u}^{s}}{\partial t}\right) d x \leq C_{1}(\varepsilon), \\
& \max _{0<t<T} \int_{Q_{f}}\left|\mathbf{u}^{f}(\mathbf{x}, t)\right|^{2} d x+\max _{0<t<T} \int_{Q_{s}}\left|\frac{\partial \mathbf{u}^{s}}{\partial t}(\mathbf{x}, t)\right|^{2} d x \leq C_{2}(\varepsilon) .
\end{aligned}
$$

Proof First of all, note that due to linearity of the problem it suffices to find corresponding a priori estimates.

To prove the first estimate in (36) we multiply the Stokes equation for $\mathbf{u}^{f}$ by $\mathbf{u}^{f}$ and integrate by parts over domain $Q_{f}$, multiply the Lamé equation for $\mathbf{u}^{s}$ by $\frac{\partial \mathbf{u}^{s}}{\partial t}$, integrate by parts over domain $Q_{s}$, and sum results.

To get the second estimate in (36) we differentiate the Stokes equation for $\mathbf{u}^{f}$ and the Lamé equation for $\mathbf{u}^{s}$ with respect to time, multiply the first expression by $\mathbf{u}^{f}$ and integrate by parts over domain $Q_{f} \times\left(0, t_{0}\right)$, multiply the second expression by $\frac{\partial \mathbf{u}^{s}}{\partial t}$ and integrate by parts over domain $Q_{s} \times\left(0, t_{0}\right)$, and sum results:

$$
\begin{aligned}
& \frac{\mu}{2} \int_{Q_{f}} \mathbb{D}\left(\mathbf{u}^{f}\left(\mathbf{x}, t_{0}\right)\right): \mathbb{D}\left(\mathbf{u}^{f}\left(\mathbf{x}, t_{0}\right)\right) d x \\
& \quad+\lambda \int_{0}^{t_{0}} \int_{Q_{s}} \mathbb{D}\left(\frac{\partial \mathbf{u}^{s}}{\partial t}(\mathbf{x}, t)\right): \mathbb{D}\left(\frac{\partial \mathbf{u}^{s}}{\partial t}(\mathbf{x}, t)\right) d x d t \\
& =\int_{0}^{t_{0}} \int_{Q_{f}} \frac{\partial \widetilde{\rho}^{\varepsilon}}{\partial t}(\mathbf{x}, t)\left(\mathbf{u}^{f}(\mathbf{x}, t) \cdot \mathbf{e}\right) d x d t \equiv I
\end{aligned}
$$


Thus,

$$
\begin{array}{rl}
\int_{Q_{f}} & \mathbb{D}\left(\mathbf{u}^{f}\left(\mathbf{x}, t_{0}\right)\right): \mathbb{D}\left(\mathbf{u}^{f}\left(\mathbf{x}, t_{0}\right)\right) d x \\
& +\int_{0}^{t_{0}} \int_{Q_{s}} \mathbb{D}\left(\frac{\partial \mathbf{u}^{s}}{\partial t}(\mathbf{x}, t)\right): \mathbb{D}\left(\frac{\partial \mathbf{u}^{s}}{\partial t}(\mathbf{x}, t)\right) d x d t \\
\leq & |I| \leq \int_{0}^{t_{0}} \int_{Q_{f}}\left|\mathbf{u}^{f}(\mathbf{x}, t)\right|^{2} d x d t+C_{0}(\varepsilon) .
\end{array}
$$

To estimate the right-hand side in (38) we introduce a new function $u(\mathbf{x}, t)$ :

$$
\mathbf{u}(\mathbf{x}, t)=\mathbf{u}^{f}(\mathbf{x}, t) \quad \text { for } \mathbf{x} \in Q_{f}, \quad \text { and } \quad \mathbf{u}(\mathbf{x}, t)=\frac{\partial \mathbf{u}^{s}}{\partial t}(\mathbf{x}, t) \quad \text { for } \mathbf{x} \in Q_{s}
$$

It is easy to see that $\mathbf{u} \in W_{2}^{1}(Q), \mathbf{u}(\mathbf{x}, t)=0$ for $\mathbf{x} \in S^{0}$ (see the boundary condition in (31)), and

$$
\begin{aligned}
& \int_{Q}|\mathbf{u}(\mathbf{x}, t)|^{2} d x=\int_{Q_{f}}\left|\mathbf{u}^{f}(\mathbf{x}, t)\right|^{2} d x+\int_{Q_{s}}\left|\frac{\partial \mathbf{u}^{s}}{\partial t}(\mathbf{x}, t)\right|^{2} d x \\
& \int_{Q} \mathbb{D}(\mathbf{u}(\mathbf{x}, t)): \mathbb{D}(\mathbf{u}(\mathbf{x}, t)) d x \\
& =\int_{Q_{f}} \mathbb{D}\left(\mathbf{u}^{f}(\mathbf{x}, t)\right): \mathbb{D}\left(\mathbf{u}^{f}(\mathbf{x}, t)\right) d x+\int_{0}^{t_{0}} \int_{Q_{s}} \mathbb{D}\left(\frac{\partial \mathbf{u}^{s}}{\partial t}(\mathbf{x}, t)\right): \mathbb{D}\left(\frac{\partial \mathbf{u}^{s}}{\partial t}(\mathbf{x}, t)\right) d x d t .
\end{aligned}
$$

Therefore, we may apply the Friedrichs-Poincaré inequality [15]

$$
\int_{Q}|\mathbf{u}(\mathbf{x}, t)|^{2} d x \leq C \int_{Q} \mathbb{D}(\mathbf{u}(\mathbf{x}, t)): \mathbb{D}(\mathbf{u}(\mathbf{x}, t)) d x,
$$

which together with (38) and (39) imply

$$
\begin{array}{rl}
\int_{Q_{f}} & \mathbb{D}\left(\mathbf{u}^{f}\left(\mathbf{x}, t_{0}\right)\right): \mathbb{D}\left(\mathbf{u}^{f}\left(\mathbf{x}, t_{0}\right)\right) d x \\
\quad \leq C \int_{0}^{t_{0}} \int_{Q_{f}} \mathbb{D}\left(\mathbf{u}^{f}(\mathbf{x}, t)\right): \mathbb{D}\left(\mathbf{u}^{f}(\mathbf{x}, t)\right) d x d t+C_{0}(\varepsilon) .
\end{array}
$$

In turn, if we put

$$
y(t)=\int_{0}^{t} \int_{Q_{f}} \mathbb{D}\left(\mathbf{u}^{f}(\mathbf{x}, \tau)\right): \mathbb{D}\left(\mathbf{u}^{f}(\mathbf{x}, \tau)\right) d x d \tau,
$$

we arrive at the differential inequality

$$
\frac{d y}{d t}(t) \leq C y(t)+C_{0}(\varepsilon), \quad y(0)=0
$$

This last equation results in

$$
\max _{0<t<T} y(t) \leq C_{1}(\varepsilon)
$$

and, consequently, the second estimate in (36) follows.

The third estimate in (36) follows from (40). 
3.1.2 Solvability of the modified problem (29), (30)

Lemma 2 Under the same conditions of Theorem 2.1 the problem (29), (30) has a unique weak solution.

Proof For given $\widetilde{\rho}$ we may find the solution $\mathbf{u}^{f}=\Phi_{1}(\widetilde{\rho})$ of the problem (33), then solve the initial boundary value problem (34) and find $\rho=\Phi_{2}\left(\mathbf{u}^{f}\right)=\Phi(\widetilde{\rho})$ such that

$$
\rho^{-} \leq \rho(\mathbf{x}, t) \leq \rho^{+}, \quad \rho \in C^{2,1}(\bar{G}) .
$$

The first estimate follows from the maximum principle and shows that $\Phi$ transforms $\mathcal{M}$ into itself, and the smoothness of $\rho$ follows from existence theorems for parabolic equations with smooth coefficients ([13], p.320).

So, if we prove the continuity of the operator $\Phi$, then $\Phi$ would be completely continuous due to (41). Finally, the Schauder fixed point theorem [14] permits us to find a fixed point of the operator $\Phi$ and solve the problem (29), (30).

The continuity of the linear operator $\Phi_{1}$

$$
\Phi_{1}: \mathcal{M} \rightarrow \mathcal{B}=L_{\infty}\left((0, T) ; W_{2}^{1}\left(Q_{f}\right)\right)
$$

follows from estimates (34).

The nonlinear operator $\Phi_{2}$ is also continuous.

In fact, let $\mathbf{u}_{1}^{f}, \mathbf{u}_{2}^{f} \in L_{\infty}\left((0, T) ; W_{2}^{1}\left(Q_{f}\right)\right)$. Then

$$
\mathbf{v}_{i}^{\varepsilon}=\mathbf{M}_{\varepsilon}^{(1)}\left(\mathbf{M}_{\varepsilon}^{(2)}\left(\mathbf{u}_{i}^{f}\right)\right) \in C^{\infty}(\bar{G}),
$$

and for the differences

$$
\rho=\rho_{1}-\rho_{2}, \quad \rho_{i}=\Phi_{2}\left(\mathbf{u}_{i}^{f}\right), \quad \mathbf{v}=\mathbf{v}_{1}^{\varepsilon}-\mathbf{v}_{2}^{\varepsilon},
$$

one has

$$
\begin{aligned}
& \frac{\partial \rho}{\partial t}+\mathbf{v}_{1}^{\varepsilon} \cdot \nabla \rho=\mathbf{v} \cdot \nabla \rho_{2}, \quad \mathbf{x} \in Q, 0<t<T, \\
& \rho(\mathbf{x}, t)=0, \quad \mathbf{x} \in S_{f}^{ \pm}, 0<t<T, \\
& \rho(\mathbf{x}, 0)=0, \quad \mathbf{x} \in Q .
\end{aligned}
$$

Therefore

$$
\left|\rho_{1}-\rho_{2}\right|_{G}^{(0)}=|\rho|_{G}^{(0)} \leq C_{1}(\varepsilon)|\mathbf{v}|_{G}^{(1)} \leq C_{2}(\varepsilon)\left\|\mathbf{u}_{1}^{f}-\mathbf{u}_{2}^{f}\right\|_{\mathcal{B}},
$$

which proves the complete continuity of the operator $\Phi$ and the solvability of the problem (29).

To prove the uniqueness of the problem (29), (30) we suppose that there exist two different solutions $\left(\mathbf{u}_{i}^{f, \varepsilon}, \mathbf{u}_{i}^{s, \varepsilon}, \rho_{i}^{\varepsilon}\right), i=1,2$.

Then the difference $\left\{\mathbf{u}^{f}, \mathbf{u}^{s}, \rho\right\}, \mathbf{u}^{f}=\mathbf{u}_{1}^{f, \varepsilon}-\mathbf{u}_{2}^{f, \varepsilon}, \mathbf{u}^{s}=\mathbf{u}_{1}^{s, \varepsilon}-\mathbf{u}_{2}^{s, \varepsilon}, \rho=\rho_{1}^{\varepsilon}-\rho_{2}^{\varepsilon}$, satisfies the following initial boundary value problem:

$$
\nabla \cdot \mathbb{P}_{f}+\rho \mathbf{e}=0, \quad \nabla \cdot \mathbf{u}^{f}=0, \quad \mathbf{x} \in Q_{f}, 0<t<T,
$$




$$
\begin{aligned}
& \nabla \cdot \mathbb{P}_{s}=0, \quad \nabla \cdot \mathbf{u}^{s}=0, \quad \mathbf{x} \in Q_{s}, 0<t<T, \quad \mathbf{u}^{s}(\mathbf{x}, 0)=0, \quad \mathbf{x} \in S, \\
& \mathbf{u}^{f}=\frac{\partial \mathbf{u}^{s}}{\partial t}, \quad \mathbb{P}_{f} \cdot \mathbf{n}=\mathbb{P}_{s} \cdot \mathbf{n}, \quad \mathbf{x} \in S, 0<t<T, \\
& \mathbb{P}_{i} \cdot \mathbf{e}_{1}=0, \quad \mathbf{x} \in S_{i}^{ \pm}, i=f, s, \quad \mathbf{u}^{s}(\mathbf{x}, t)=0, \quad \mathbf{x} \in S^{0}, 0<t<T, \\
& \frac{\partial \rho}{\partial t}+\mathbf{v}_{1}^{\varepsilon} \cdot \nabla \rho=\mathbf{v}^{\varepsilon} \cdot \nabla \rho_{2}^{\varepsilon}, \quad \mathbf{x} \in Q, 0<t<T, \\
& \rho(\mathbf{x}, t)=0, \quad \mathbf{x} \in S_{f}^{ \pm}, 0<t<T, \\
& \rho(\mathbf{x}, 0)=0, \quad \mathbf{x} \in Q, \\
& \mathbf{v}^{\varepsilon}=\mathbf{M}_{\varepsilon}^{(1)}\left(\mathbf{M}_{\varepsilon}^{(2)}(\mathbf{u})\right), \quad \mathbb{P}_{f}=2 \mu \mathbb{D}\left(\mathbf{u}^{f}\right)-p_{f} \mathbb{I}, \quad \mathbb{P}_{s}=2 \lambda \mathbb{D}\left(\mathbf{u}^{s}\right)-p_{s} \mathbb{I} .
\end{aligned}
$$

Now we multiply the dynamic equation for $\mathbf{u}^{f}$ by $\mathbf{u}^{f}$ and integrate by parts over domain $Q_{f} \times\left(0, t_{0}\right)$, the dynamic equation for $\mathbf{u}^{s}$ by $\frac{\partial \mathbf{u}^{s}}{\partial t}$ and integrate by parts over domain $Q_{s} \times$ $\left(0, t_{0}\right)$ the equation for $\rho$ by $\rho$ and integrate by parts over domain $Q_{f} \times\left(0, t_{0}\right)$, and sum all results:

$$
\begin{aligned}
& \mu \int_{0}^{t_{0}} \int_{Q_{f}} \mathbb{D}\left(\mathbf{u}^{f}(\mathbf{x}, t)\right): \mathbb{D}\left(\mathbf{u}^{f}(\mathbf{x}, t)\right) d x d t \\
& \quad+\frac{1}{2} \int_{Q_{f}}\left|\rho\left(\mathbf{x}, t_{0}\right)\right|^{2} d x+\frac{\lambda}{2} \int_{Q_{s}} \mathbb{D}\left(\mathbf{u}^{s}\left(\mathbf{x}, t_{0}\right)\right): \mathbb{D}\left(\mathbf{u}^{s}\left(\mathbf{x}, t_{0}\right)\right) d x \\
& \quad \int_{0}^{t_{0}} \int_{Q_{f}} \rho(\mathbf{x}, t)\left(\mathbf{u}^{f}(\mathbf{x}, t) \mathbf{e}\right) d x d t \equiv I_{0} .
\end{aligned}
$$

Introducing the new function

$$
\begin{aligned}
& \mathbf{w}(\mathbf{x}, t)=\int_{0}^{t} \mathbf{u}^{f}(\mathbf{x}, \tau) d \tau, \quad \mathbf{x} \in Q_{f}, \quad \mathbf{w}(\mathbf{x}, t)=\mathbf{u}^{s}(\mathbf{x}, t), \quad \mathbf{x} \in Q_{s}, \\
& \mathbf{u}(\mathbf{x}, t)=0, \quad \mathbf{x} \in S^{0}
\end{aligned}
$$

in the same way as before (see estimates (36)) we get

$$
\left|I_{0}\right| \leq \delta \int_{0}^{t_{0}} \int_{Q_{f}}\left|\mathbf{u}^{f}(\mathbf{x}, t)\right|^{2} d x d t+C(\delta) \int_{0}^{t_{0}} \int_{Q_{f}}|\rho(\mathbf{x}, t)|^{2} d x d t
$$

for arbitrary small $\delta>0$, and

$$
\int_{Q_{f}}\left|\rho\left(\mathbf{x}, t_{0}\right)\right|^{2} d x \leq C(\delta) \int_{0}^{t_{0}} \int_{Q_{f}}|\rho(\mathbf{x}, t)|^{2} d x d t, \quad \int_{Q_{f}}|\rho(\mathbf{x}, 0)|^{2} d x=0 .
$$

The Gronwall inequality results in $\rho(\mathbf{x}, t)=0$ almost everywhere in $G$. 


\subsection{Uniform bounds for the solutions of the problem (29), (30)}

\subsection{1 $L_{2}$-Estimates for the solutions of the problem (29), (30)}

Lemma 3 Under the conditions of Theorem 2.1 for the solution $\mathbf{u}^{\varepsilon}$ of the problem (29) one gets the following estimates:

$$
\begin{aligned}
& \int_{0}^{T} \int_{Q_{f}} \mathbb{D}\left(\mathbf{u}^{f, \varepsilon}\right): \mathbb{D}\left(\mathbf{u}^{f, \varepsilon}\right) d x d t+\max _{0<t<T} \int_{Q_{s}} \mathbb{D}\left(\mathbf{u}^{s, \varepsilon}(\mathbf{x}, t)\right): \mathbb{D}\left(\mathbf{u}^{s, \varepsilon}(\mathbf{x}, t)\right) d x \leq C, \\
& \max _{0<t<T} \int_{Q_{f}} \mathbb{D}\left(\mathbf{u}^{f, \varepsilon}(\mathbf{x}, t)\right): \mathbb{D}\left(\mathbf{u}^{f, \varepsilon}(\mathbf{x}, t)\right) d x+\int_{0}^{T} \int_{Q_{s}} \mathbb{D}\left(\frac{\partial \mathbf{u}^{s, \varepsilon}}{\partial t}\right): \mathbb{D}\left(\frac{\partial \mathbf{u}^{s, \varepsilon}}{\partial t}\right) d x \leq C, \\
& \max _{0<t<T} \int_{Q_{f}}\left|\mathbf{u}^{f, \varepsilon}(\mathbf{x}, t)\right|^{2} d x+\max _{0<t<T} \int_{Q_{s}}\left|\frac{\partial \mathbf{u}^{s, \varepsilon}}{\partial t}(\mathbf{x}, t)\right|^{2} d x \leq C .
\end{aligned}
$$

Proof The proof of these estimates almost exactly repeats the proof of estimates (36). The single difference is in the estimation of the term $I$ in (37):

$$
\begin{aligned}
& \frac{\mu}{2} \int_{Q_{f}} \mathbb{D}\left(\mathbf{u}^{f, \varepsilon}\left(\mathbf{x}, t_{0}\right)\right): \mathbb{D}\left(\mathbf{u}^{f, \varepsilon}\left(\mathbf{x}, t_{0}\right)\right) d x \\
& \quad+\lambda \int_{0}^{t_{0}} \int_{Q_{s}} \mathbb{D}\left(\frac{\partial \mathbf{u}^{s, \varepsilon}}{\partial t}(\mathbf{x}, t)\right): \mathbb{D}\left(\frac{\partial \mathbf{u}^{s, \varepsilon}}{\partial t}(\mathbf{x}, t)\right) d x d t \\
& =\int_{0}^{t_{0}} \int_{Q_{f}} \frac{\partial \rho^{\varepsilon}}{\partial t}(\mathbf{x}, t)\left(\mathbf{u}^{f, \varepsilon}(\mathbf{x}, t) \cdot \mathbf{e}\right) d x d t \equiv I^{\varepsilon} .
\end{aligned}
$$

To estimate $I^{\varepsilon}$ we use the differential equation for $\rho^{\varepsilon}$ in (30):

$$
\begin{aligned}
\left|I^{\varepsilon}\right| & =\left|\int_{0}^{t_{0}} \int_{Q_{f}}\left(\mathbf{u}^{f, \varepsilon} \cdot \mathbf{e}\right) \nabla \cdot\left(\rho^{\varepsilon} \mathbf{v}^{\varepsilon}\right) d x d t\right| \\
& =\left|\int_{0}^{t_{0}} \int_{Q_{f}}\left(\rho^{\varepsilon} \mathbf{v}^{\varepsilon}\right) \cdot \nabla \mathbf{u}^{f, \varepsilon} \cdot \mathbf{e} d x d t\right| \\
& \leq \int_{0}^{t_{0}} \int_{Q_{f}} \mathbb{D}\left(\mathbf{u}^{f, \varepsilon}(\mathbf{x}, t)\right): \mathbb{D}\left(\mathbf{u}^{f, \varepsilon}(\mathbf{x}, t)\right) d x d t+C \int_{0}^{t_{0}} \int_{Q_{f}}\left(\mathbf{u}^{f, \varepsilon}(\mathbf{x}, t)\right)^{2} d x d t+C,
\end{aligned}
$$

where we have used the evident estimates for the mollifiers $\mathbf{M}_{\varepsilon}^{(1)}\left(\mathbf{M}_{\varepsilon}^{(2)}\right)$

$$
\int_{0}^{t_{0}} \int_{Q_{f}}\left(\mathbf{v}^{\varepsilon}(\mathbf{x}, t)\right)^{2} d x d t \leq C \int_{0}^{t_{0}} \int_{Q_{f}}\left(\mathbf{u}^{f, \varepsilon}(\mathbf{x}, t)\right)^{2} d x d t .
$$

The rest of the proof is the same as for (36).

Lemma 4 Under the conditions of Theorem 2.1 let $\mathbf{u}^{f, \varepsilon}$ be the solution of the problem (29). Then $\mathbb{P}_{f}\left(\mathbf{u}^{f, \varepsilon}, p_{f}^{\varepsilon}\right) \in L_{\infty}\left((0, T) ; L_{2}\left(Q_{f}\right)\right)$,

$$
\max _{0<t<T} \int_{Q_{f}}\left|p_{f}^{\varepsilon}(\mathbf{x}, t)\right|^{2} d x \leq C,
$$




$$
\begin{array}{r}
\mathbb{P}_{s}\left(\mathbf{u}^{s, \varepsilon}, p_{s}^{\varepsilon}\right) \in L_{\infty}\left((0, T) ; L_{2}\left(Q_{s}\right)\right), \\
\max _{0<t<T} \int_{Q_{s}}\left|p_{s}^{\varepsilon}(\mathbf{x}, t)\right|^{2} d x \leq C,
\end{array}
$$

and for any $\Omega_{f} \subset Q_{f}$ and $\Omega_{s} \subset Q_{s}$

$$
\mathbf{u}^{f, \varepsilon} \in L_{\infty}\left((0, T) ; W_{m}^{2}\left(\Omega_{f}\right)\right), \quad \mathbf{u}^{s, \varepsilon} \in L_{\infty}\left((0, T) ; W_{m}^{2}\left(\Omega_{s}\right)\right)
$$

for all $m>2$.

Proof Let $\varphi \in \stackrel{\circ}{W}_{2}^{1}\left(Q_{f}\right)$ be a test function in the integral identity (35). Then this identity takes the form

$$
\int_{Q_{f}} p_{f}^{\varepsilon} \nabla \cdot \boldsymbol{\varphi} d x=\int_{Q_{f}}\left(2 \mu \mathbb{D}\left(\mathbf{u}^{f, \varepsilon}\right): \mathbb{D}(\boldsymbol{\varphi})-\left(\mathbf{f}+\rho^{\varepsilon} \mathbf{e}\right) \cdot \boldsymbol{\varphi}\right) d x .
$$

Now we choose $\varphi$ as a solution of the problem

$$
\begin{aligned}
& \boldsymbol{\varphi}=\boldsymbol{\varphi}_{0}+\nabla \psi, \\
& \Delta \psi=p_{f}^{\varepsilon}, \quad \mathbf{x} \in Q_{f}, \quad \psi(\mathbf{x}, t)=0, \quad \mathbf{x} \in S, 0<t<T, \\
& \nabla \cdot \boldsymbol{\varphi}_{0}=0, \quad \mathbf{x} \in Q_{f}, \quad \nabla \psi(\mathbf{x}, t)+\boldsymbol{\varphi}_{0}(\mathbf{x}, t)=0, \quad \mathbf{x} \in S, 0<t<T .
\end{aligned}
$$

The above problem has a unique solution [16] and

$$
\max _{0<t<T} \int_{Q_{f}}|\nabla \boldsymbol{\varphi}(\mathbf{x}, t)|^{2} d x \leq C\left\|p_{f}^{\varepsilon}\right\|_{2, Q_{f}}^{2} .
$$

Thus (44), (47), and (48) result in

$$
\max _{0<t<T} \int_{Q_{f}}\left|p_{f}^{\varepsilon}(\mathbf{x}, t)\right|^{2} d x \leq C,
$$

and $\mathbb{P}_{f}\left(\mathbf{u}^{f, \varepsilon}, p_{f}^{\varepsilon}\right) \in L_{\infty}\left((0, T) ; L_{2}\left(Q_{f}\right)\right)$.

Coming back to (47) we conclude that $\mathbb{P}_{f}\left(\mathbf{u}^{f, \varepsilon}, p_{f}^{\varepsilon}\right) \in L_{\infty}\left((0, T) ; W_{2}^{1}\left(Q_{f}\right)\right)$, and (47) is equivalent to the Stokes equation

$$
\mu \Delta \mathbf{u}^{f, \varepsilon}-\nabla p_{f}^{\varepsilon}+\mathbf{f}+\rho^{\varepsilon} \mathbf{e}=0, \quad \mathbf{x} \in Q_{f}, 0<t<T
$$

The right-hand side $\mathbf{F}=\mathbf{f}+\rho^{\varepsilon} \mathbf{e}$ of the differential equation belongs to $L_{\infty}(G)$. Therefore we may use the same arguments as in [1] and conclude that for any $\Omega \subset Q_{f}$

$$
\mathbf{u}^{f, \varepsilon} \in L_{\infty}\left((0, T) ; W_{m}^{2}(\Omega)\right) \text { for any } m>2 \text {. }
$$

Now we apply the same arguments for the solid component:

$$
\max _{0<t<T} \int_{Q_{s}}\left|p_{s}^{\varepsilon}(\mathbf{x}, t)\right|^{2} d x \leq C,
$$

and $\mathbb{P}_{s}\left(\mathbf{u}^{s, \varepsilon}, p_{s}^{\varepsilon}\right) \in L_{\infty}\left((0, T) ; L_{2}\left(Q_{s}\right)\right)$. 
3.2.2 Uniform estimates for the solutions of the problem (29), (30) in Hölder's spaces

Lemma 5 Under the conditions of Theorem 2.1 let $\mathbf{u}^{f, \varepsilon}$ be the solution of the problem (29).

Then

$$
\max _{0<t<T}\left|\mathbf{u}^{f, \varepsilon}(\cdot, t)\right|_{Q_{f}^{(2 \delta)}}^{(1+\alpha)} \leq C(\alpha, \delta)
$$

for $0<\delta<\delta_{0}$ with sufficiently small $\delta_{0}$, and any $\alpha, 0<\alpha<1$.

Proof The domain $Q_{f}^{(\delta)}$ consists of two disconnected parts. For each part the proof is the same. Note also that

$$
\max _{0<t<T}\left|\mathbf{u}^{f, \varepsilon}(\cdot, t)\right|_{\Omega(f, \delta)}^{(1+\alpha)} \leq C(\alpha, \delta)
$$

for any domain $\Omega^{(f, \delta)} \subset Q_{f}^{(\delta)}$ with the distance to the solid part $Q_{s}$ greater than $\delta$.

So, we may restrict ourselves only to the part in $x_{2}<0$ and the following domains $\Omega^{(\delta)}$. For $0<\delta<\frac{1}{2}$ we put

$$
\begin{aligned}
& \Omega^{(\delta)}=\left\{\mathbf{x} \in Q:-1+\delta<x_{1}<1-\delta,-\frac{1}{2}+\delta<x_{2}<-\frac{1}{2}-\delta\right\}, \\
& \Omega_{f}^{(\delta)}=\Omega^{(\delta)} \cap Q_{f} .
\end{aligned}
$$

Let $\zeta^{(\delta)}(\mathbf{x})$ be infinitely smooth functions such that $\zeta^{(\delta)}(\mathbf{x})=1$ for $\mathbf{x} \in \Omega^{(2 \delta)}$, and $\zeta^{(\delta)}(\mathbf{x})=0$ for $\mathbf{x} \in Q \backslash \Omega^{(\delta)}$.

The functions $\mathbf{u}^{f, \varepsilon, \delta}=\mathbf{u}^{f, \varepsilon} \zeta^{(\delta)}, p_{f}^{\varepsilon, \delta}=p_{f}^{\varepsilon} \zeta^{(\delta)}, \mathbf{u}^{s, \varepsilon, \delta}=\mathbf{u}^{s, \varepsilon} \zeta^{(\delta)}$, and $p_{s}^{\varepsilon, \delta}=p_{s}^{\varepsilon} \zeta^{(\delta)}$ satisfy in $\mathbb{R}^{2}$ for $t>0$ the following linear problem:

$$
\begin{aligned}
& \mu \triangle \mathbf{u}^{f, \varepsilon, \delta}-\nabla p_{f}^{\varepsilon, \delta}=\mathbf{F}^{\varepsilon, \delta}, \quad \nabla \cdot \mathbf{u}^{f, \varepsilon, \delta}=\varphi^{\varepsilon, \delta}, \quad x_{2}>-\frac{1}{2}, 0<t<T ; \\
& \lambda \triangle \mathbf{u}^{s, \varepsilon, \delta}-\nabla p_{s}^{\varepsilon, \delta}=\mathbf{F}^{\varepsilon, \delta}, \quad \nabla \cdot \mathbf{u}^{s, \varepsilon, \delta}=\varphi^{\varepsilon, \delta}, \quad x_{2}<-\frac{1}{2}, 0<t<T ; \\
& \mu\left(\frac{\partial}{\partial x_{2}} u_{1}^{f, \varepsilon, \delta}+\frac{\partial}{\partial x_{1}} u_{2}^{f, \varepsilon, \delta}\right)=\lambda\left(\frac{\partial}{\partial x_{2}} u_{1}^{s, \varepsilon, \delta}+\frac{\partial}{\partial x_{1}} u_{2}^{s, \varepsilon, \delta}\right)+\psi_{1}, \\
& \mu \frac{\partial}{\partial x_{2}} u_{2}^{f, \varepsilon, \delta}+p_{f}^{\varepsilon, \delta}=\lambda \frac{\partial}{\partial x_{2}} u_{2}^{s, \varepsilon, \delta}+p_{s}^{\varepsilon, \delta}+\psi_{2}, \\
& u_{1}^{f, \varepsilon, \delta}=\frac{\partial}{\partial t} u_{1}^{s, \varepsilon, \delta}, \quad u_{2}^{f, \varepsilon, \delta}=\frac{\partial}{\partial t} u_{2}^{s, \varepsilon, \delta}, \quad x_{2}=\frac{1}{2}, 0<t<T, \\
& \mathbf{u}^{s, \varepsilon, \delta}(\mathbf{x}, 0)=0, \quad x_{2}=-\frac{1}{2} .
\end{aligned}
$$


Here

$$
\begin{aligned}
& \mathbf{F}^{\varepsilon, \delta}=\zeta^{(\delta)}\left(\rho^{\varepsilon} \mathbf{e}+\mathbf{f}\right)+\mu \nabla \mathbf{u}^{f, \varepsilon, \delta} \cdot \nabla \zeta^{(\delta)}+p_{f}^{\varepsilon, \delta} \nabla \zeta^{(\delta)} \\
& \varphi^{\varepsilon, \delta}=\mathbf{u}^{f, \varepsilon, \delta} \cdot \nabla \zeta^{(\delta)}, \quad x_{2}>-\frac{1}{2} ; \\
& \psi_{1}=\frac{\partial \zeta^{(\delta)}}{\partial x_{1}}\left(\mu u_{2}^{f, \varepsilon, \delta}-\lambda u_{2}^{s, \varepsilon, \delta}\right)+\frac{\partial \zeta^{(\delta)}}{\partial x_{2}}\left(\mu u_{1}^{f, \varepsilon, \delta}-\lambda u_{1}^{s, \varepsilon, \delta}\right), \\
& \psi_{2}=\frac{\partial \zeta^{(\delta)}}{\partial x_{2}}\left(\mu u_{2}^{f, \varepsilon, \delta}-\lambda u_{2}^{s, \varepsilon, \delta}\right), \quad x_{2}=-\frac{1}{2} ; \\
& \mathbf{F}^{\varepsilon, \delta}=\zeta^{(\delta)} \mathbf{f}+\lambda \nabla \mathbf{u}^{s, \varepsilon, \delta} \cdot \nabla \zeta^{(\delta)}+p_{s}^{\varepsilon, \delta} \nabla \zeta^{(\delta)}, \quad \varphi^{\varepsilon, \delta}=\mathbf{u}^{s, \varepsilon, \delta} \cdot \nabla \zeta^{(\delta)} x_{2}<-\frac{1}{2} ; \\
& \max _{0<t<T}\left\|\mathbf{F}^{\varepsilon, \delta}(\cdot, t)\right\|_{2, \mathbb{R}^{2}}+\max _{0<t<T}\left\|\varphi^{\varepsilon, \delta}(\cdot, t)\right\|_{2, \mathbb{R}^{2}}^{(1)} \leq C(\delta) .
\end{aligned}
$$

For the sake of simplicity we denote all constants independent of $\varepsilon$ as $C$ (or $C(\delta)$ ), and omit for the moment the indices $\varepsilon$ and $\delta$.

Now we reduce (53) to homogeneous differential equations by introducing new functions $\left\{\mathbf{w}^{f}, r_{f}, \mathbf{w}^{s}, r_{s}\right\}$ as a solution to the following problem:

$$
\begin{aligned}
& \mu \Delta \mathbf{w}^{f}-\nabla r_{f}=\mathbf{F}, \quad \nabla \cdot \mathbf{w}^{f}=\varphi, \quad x_{2}+\frac{1}{2}>0,0<t<T ; \\
& \lambda \triangle \mathbf{w}^{s}-\nabla r_{s}=\mathbf{F}, \quad \nabla \cdot \mathbf{w}^{s}=\varphi, \quad x_{2}+\frac{1}{2}<0,0<t<T ; \\
& \mu\left(\frac{\partial w_{1}^{f}}{\partial x_{2}}+\frac{\partial w_{2}^{f}}{\partial x_{1}}\right)=\lambda\left(\frac{\partial w_{1}^{s}}{\partial x_{2}}+\frac{\partial w_{2}^{s}}{\partial x_{1}}\right)+\psi_{1}, \\
& \mu \frac{\partial w_{2}^{f}}{\partial x_{2}}+r_{f}=\lambda \frac{\partial w_{2}^{s}}{\partial x_{2}}+r_{s}+\psi_{2}, \\
& w_{1}^{f}=\frac{\partial w_{1}^{s}}{\partial t}, \quad w_{2}^{f}=\frac{\partial w_{2}^{s}}{\partial t}, \quad x_{2}=-\frac{1}{2}, 0<t<T, \quad \mathbf{w}^{s}(\mathbf{x}, 0)=0, \quad x_{2}=-\frac{1}{2} .
\end{aligned}
$$

Thus, for

$$
\mathbf{v}=\mathbf{u}-\mathbf{w}, \quad q=p-r
$$

one has

$$
\begin{aligned}
& \mu \Delta \mathbf{v}^{f}-\nabla q_{f}=0, \quad \nabla \cdot \mathbf{v}^{f}=0, \quad x_{2}+\frac{1}{2}>0,0<t<T ; \\
& \lambda \Delta \mathbf{v}^{s}-\nabla q_{s}=0, \quad \nabla \cdot \mathbf{v}^{s}=0, \quad x_{2}+\frac{1}{2}<0,0<t<T ; \\
& \mu\left(\frac{\partial v_{1}^{f}}{\partial x_{2}}+\frac{\partial v_{2}^{f}}{\partial x_{1}}\right)=\lambda\left(\frac{\partial v_{1}^{s}}{\partial x_{2}}+\frac{\partial v_{2}^{s}}{\partial x_{1}}\right), \\
& \mu \frac{\partial v_{2}^{f}}{\partial x_{2}}+q_{f}=\lambda \frac{\partial v_{2}^{s}}{\partial x_{2}}+q_{s}, \\
& \frac{\partial v_{1}^{s}}{\partial t}=v_{1}^{f}+\varphi_{1}, \quad \frac{\partial v_{2}^{s}}{\partial t}=v_{2}^{f}+\varphi_{2}, \quad x_{2}=-\frac{1}{2}, 0<t<T, \\
& \mathbf{v}^{s}(\mathbf{x}, 0)=0, \quad x_{2}=-\frac{1}{2} .
\end{aligned}
$$


Here

$$
\boldsymbol{\varphi}\left(x_{1}, t\right)=\left(\varphi_{1}\left(x_{1}, t\right), \varphi_{2}\left(x_{1}, t\right)\right)=\mathbf{w}^{f}\left(x_{1}, 0, t\right)-\frac{\partial \mathbf{w}^{s}}{\partial t}\left(x_{1}, 0, t\right) .
$$

Note that due to the homogeneous boundary condition in (55) for $\mathbf{w}^{s}$ at $S$

$$
\frac{\partial \mathbf{w}^{s}}{\partial t}\left(x_{1}, 0, t\right) \equiv 0 \quad \text { and } \quad \varphi_{i}\left(x_{1}, t\right)=w_{i}^{f}\left(x_{1}, 0, t\right) \quad \text { for } i=1,2 \text { and } t>0 \text {. }
$$

To solve (56) we apply the Fourier transform

$$
\widehat{v}\left(\xi, x_{2}, t\right)=\frac{1}{\sqrt{2 \pi}} \int_{-\infty}^{\infty} v\left(x_{1}, x_{2}, t\right) e^{-i \xi x_{1}} d x_{1}
$$

with respect the variable $x_{1}$, and we get the following system of ordinary differential equations in the variable $x_{2}$ :

$$
\begin{aligned}
& \mu \frac{\partial^{2} \widehat{v}_{1}^{f}}{\partial x^{2}}-\mu \xi^{2} \widehat{v}_{1}^{f}+i \xi \widehat{p}_{f}=0, \\
& \mu \frac{\partial^{2} \widehat{v}_{2}^{f}}{\partial x^{2}}-\mu \xi^{2} \widehat{v}_{2}^{f}-\frac{\partial \widehat{p}_{f}}{\partial x_{2}}=0, \quad \frac{\partial^{2} \widehat{p}_{f}}{\partial x^{2}}-\xi^{2} \widehat{p}_{f}=0, \\
& \widehat{v}_{1}^{f}=-\frac{i}{\xi} \frac{\partial \widehat{v}_{2}^{f}}{\partial x_{2}}, \quad x_{2}+\frac{1}{2}>0 \text {, } \\
& \lambda \frac{\partial^{2} \widehat{v}_{1}^{s}}{\partial x^{2}}-\lambda \xi^{2} \widehat{v}_{1}^{s}+i \xi \widehat{p}_{f}=0, \\
& \lambda \frac{\partial^{2} \widehat{v}_{2}^{s}}{\partial x^{2}}-\lambda \xi^{2} \widehat{v}_{2}^{s}-\frac{\partial \widehat{p}_{s}}{\partial x_{2}}=0, \quad \frac{\partial^{2} \widehat{p}_{s}}{\partial x^{2}}-\xi^{2} \widehat{p}_{s}=0, \\
& \widehat{v}_{1}^{s}=-\frac{i}{\xi} \frac{\partial \widehat{v}_{2}^{s}}{\partial x_{2}}, \quad x_{2}+\frac{1}{2}<0, \\
& \mu \frac{\partial \widehat{v}_{1}^{f}}{\partial x_{2}}-\mu i \xi \widehat{v}_{2}^{f}=\lambda \frac{\partial \widehat{v}_{1}^{s}}{\partial x_{2}}-\lambda i \xi \widehat{v}_{2}^{s}, \\
& \mu \frac{\partial \widehat{v}_{2}^{f}}{\partial x_{2}}+\widehat{p}_{f}=\lambda \frac{\partial \widehat{v}_{2}^{s}}{\partial x_{2}}+\widehat{p}_{s} \\
& \widehat{v}_{1}^{f}+\widehat{\varphi}_{1}=\frac{\partial \widehat{v}_{1}^{s}}{\partial t}, \quad \widehat{v}_{2}^{f}+\widehat{\varphi}_{2}=\frac{\partial \widehat{v}_{2}^{s}}{\partial t}, \quad x_{2}=-\frac{1}{2}, \\
& \widehat{v}_{1}^{s}\left(\xi, x_{2}, 0\right)=\widehat{v}_{2}^{S}\left(\xi, x_{2}, 0\right)=0 \text {. }
\end{aligned}
$$

Solutions of (57) and (58) have a very simple form:

$$
\begin{aligned}
& \widehat{p}_{f}=c_{p}^{f} e^{-|\xi| z}, \quad \widehat{v}_{1}^{f}=i\left(\frac{|\xi|}{\xi} c_{v}^{f}-\frac{1}{2 \mu \xi}(1-z|\xi|) c_{p}^{f}\right) e^{-|\xi| z} \\
& \widehat{v}_{2}^{f}=\left(c_{v}^{f}+\frac{z}{2 \mu} c_{p}^{f}\right) e^{-|\xi| z}
\end{aligned}
$$


where $z=\left|x_{2}+\frac{1}{2}\right|$,

$$
\begin{aligned}
& \widehat{p}_{s}=c_{p}^{s} e^{|\xi| z}, \quad \widehat{v}_{1}^{s}=-i\left(\frac{|\xi|}{\xi} c_{v}^{s}+\frac{1}{2 \lambda \xi}(1+z|\xi|) c_{p}^{s}\right) e^{|\xi| z} \\
& \widehat{v}_{2}^{s}=\left(c_{v}^{f}+\frac{z}{2 \mu} c_{p}^{f}\right) e^{-|\xi| z}
\end{aligned}
$$

To define the functions $c_{p}^{f}, c_{v}^{f}, c_{p}^{s}$, and $c_{v}^{s}$, we use the boundary conditions (59):

$$
\begin{aligned}
& c_{v}^{s}+\frac{1}{2 \lambda|\xi|} c_{p}^{s}=\frac{\mu}{\lambda} c_{v}^{f}-\frac{1}{2 \lambda|\xi|} c_{p}^{f}, \\
& c_{v}^{s}+\frac{3}{2 \lambda|\xi|} c_{p}^{s}=-\frac{\mu}{\lambda} c_{v}^{f}+\frac{3}{2 \lambda|\xi|} c_{p}^{f}, \\
& \frac{\partial c_{v}^{s}}{\partial t}+\frac{1}{2 \lambda|\xi|} \frac{\partial c_{p}^{s}}{\partial t}=-c_{v}^{f}+\frac{1}{2 \mu|\xi|} c_{p}^{f}+i \frac{|\xi|}{\xi} \widehat{\varphi}_{1}, \\
& \frac{\partial c_{v}^{s}}{\partial t}=c_{v}^{f}+\widehat{\varphi}_{2}, \quad c_{v}^{s}(\xi, 0)=c_{p}^{s}(\xi, 0)=0 .
\end{aligned}
$$

The first system (62) gives us values $c_{v}^{s}$ and $c_{p}^{s}$ as a combination of $c_{v}^{f}$ and $c_{p}^{f}$ :

$$
c_{v}^{s}=2 \frac{\mu}{\lambda} c_{v}^{f}-\frac{3}{2 \lambda|\xi|} c_{p}^{f}, \quad c_{p}^{s}=-2 \mu|\xi| c_{v}^{f}+2 c_{p}^{f} .
$$

Taking into account (63) and (64) we define $c_{v}^{-}$and $c_{r}^{-}$from the Cauchy problem for the following system of ordinary differential equations:

$$
\begin{aligned}
& \frac{\partial c_{v}^{f}}{\partial t}=-4 \frac{\lambda}{\mu} c_{v}^{f}+\frac{3 \lambda}{4 \mu^{2}|\xi|} c_{p}^{f}+3 i \frac{\lambda|\xi|}{\mu \xi} \widehat{\varphi}_{1}-\frac{\lambda}{\mu} \widehat{\varphi}_{2}, \\
& \frac{\partial c_{p}^{f}}{\partial t}=-6 \lambda|\xi| c_{v}^{f}+2 \frac{\lambda}{\mu} c_{p}^{f}+4 i \xi \widehat{\varphi}_{1}-2 \lambda|\xi| \widehat{\varphi}_{2}, \\
& c_{\nu}^{f}(\xi, 0)=c_{p}^{f}(\xi, 0)=0 .
\end{aligned}
$$

The last equation is equivalent to the Cauchy problem for the second order ordinary differential equation with coefficient $k^{2}=32 \frac{\lambda}{\mu}$ independent of $\xi$ :

$$
\begin{aligned}
& \frac{\partial^{2} c_{v}^{f}}{\partial t^{2}}-k^{2} c_{v}^{f}=3 \frac{\lambda}{\mu} \widehat{\varphi}_{2}+\frac{\partial \widehat{\varphi}_{1}}{\partial t}-4 \frac{\lambda}{\mu} \widehat{\varphi}_{1}, \\
& c_{v}^{f}(\xi, 0)=0, \quad \frac{\partial c_{v}^{f}}{\partial t}(\xi, 0)=3 i \frac{\lambda|\xi|}{\mu \xi} \widehat{\varphi}_{1}(\xi, 0)-\frac{\lambda}{\mu} \widehat{\varphi}_{2}(\xi, 0) .
\end{aligned}
$$

Thus,

$$
c_{l}^{j}(\xi, t)=\sum_{m=f, s} \sum_{i=1}^{2}\left(Z_{l, i}^{j, m}(t) \widehat{w}_{i}^{m}(\xi, 0,0)+\int_{0}^{t} G_{l, i}^{j, m}(t-\tau) \widehat{w}_{i}^{m}(\xi, 0, \tau) d \tau\right)
$$

for $j=f, s$ and $l=v, p$. 
Note that $\widehat{w}_{i}^{k}(\xi, 0,0), z_{l}^{j, i}$ and $g_{l}^{j, i}$ are known functions, and the functions $z_{l}^{j, i}$ and $g_{l}^{j, i}$ are infinitely smooth in $t$.

Gathering all these issues one has for $j=f, s$ and $l=1,2$

$$
\begin{aligned}
\widehat{v}_{l}^{j}(\xi, z, t)= & \sum_{m=f, s} \sum_{i=1}^{2}\left\{\left(\left(Z_{l, i, 0}^{j, m}(z, t)+\frac{|\xi|}{\xi} Z_{l, i, 1}^{j, m}(z, t)\right) \widehat{w}_{i}^{m}(\xi, 0,0)\right) e^{-|\xi| z}\right. \\
& \left.+\left(\int_{0}^{t}\left(G_{l, i, 0}^{j, m}(z, t-\tau)+\frac{|\xi|}{\xi} G_{l, i, 1}^{j, m}(z, t-\tau)\right) \widehat{w}_{i}^{m}(\xi, 0, \tau) d \tau\right)\right\} e^{-|\xi| z},
\end{aligned}
$$

where $Z_{l, i, k}^{j, m}(z, t)$ and $G_{l, i, k}^{j, m}(z, t), k=0,1$, are linear in $z \geq 0$ and infinitely smooth in $t$.

For $z=0$ we get

$$
\begin{aligned}
\widehat{v}_{l}^{j}(\xi, 0, t)= & \sum_{m=f, s} \sum_{i=1}^{2}\left\{\left(\left(Z_{l, i, 0}^{j, m}(0, t)+\frac{|\xi|}{\xi} Z_{l, i, 1}^{j, m}(0, t)\right) \widehat{w}_{i}^{m}(\xi, 0,0)\right)\right. \\
& \left.+\left(\int_{0}^{t}\left(G_{l, i, 0}^{j, m}(0, t-\tau)+\frac{|\xi|}{\xi} G_{l, i, 1}^{j, m}(0, t-\tau)\right) \widehat{w}_{i}^{m}(\xi, 0, \tau) d \tau\right)\right\}
\end{aligned}
$$

and

$$
\max _{0<t<T}\left\|\widehat{\mathbf{v}}^{j}(\cdot, 0, t)\right\|_{2, \mathbb{R}}^{\left(l-\frac{1}{2}\right)} \leq C_{0}\left(1+\sum_{m=f, s} \max _{0<t<T}\left\|\widehat{\mathbf{w}}^{m}(\cdot, 0, t)\right\|_{2, \mathbb{R}}^{\left(l-\frac{1}{2}\right)}\right) .
$$

Due to the Parseval equality

$$
\begin{aligned}
\max _{0<t<T}\left\|\mathbf{v}^{j}(\cdot, 0, t)\right\|_{2, \mathbb{R}}^{\left(l-\frac{1}{2}\right)} & =\max _{0<t<T}\left\|\widehat{\mathbf{v}}^{j}(\cdot, 0, t)\right\|_{2, \mathbb{R}}^{\left(l-\frac{1}{2}\right)} \\
& \leq C_{0}\left(1+\sum_{m=f, s} \max _{0<t<T}\left\|\widehat{\mathbf{w}}^{m}(\cdot, 0, t)\right\|_{2, \mathbb{R}}^{\left(l-\frac{1}{2}\right)}\right) \\
& =C_{0}\left(1+\sum_{m=f, s} \max _{0<t<T}\left\|\mathbf{w}^{m}(\cdot, 0, t)\right\|_{2, \mathbb{R}}^{\left(l-\frac{1}{2}\right)}\right) .
\end{aligned}
$$

Therefore (see (24))

$$
\begin{aligned}
\max _{0<t<T}\left\|\mathbf{v}^{j}(\cdot, t)\right\|_{2, \mathbb{R}_{j}^{2}}^{(l)} & \leq C_{2} \max _{0<t<T}\left\|\mathbf{v}^{j}(\cdot, t)\right\|_{2, \mathbb{R}}^{\left(l-\frac{1}{2}\right)} \leq C_{2} C_{0}\left(1+\sum_{m=f, s} \max _{0<t<T}\left\|\mathbf{w}^{m}(\cdot, 0, t)\right\|_{2, \mathbb{R}}^{\left(l-\frac{1}{2}\right)}\right) \\
& \leq C_{2} C_{0}\left(1+C_{1} \sum_{m=f, s} \max _{0<t<T}\left\|\mathbf{w}^{m}(\cdot, t)\right\|_{2, \mathbb{R}_{j}^{2}}^{(l)}\right), \quad j=f, s .
\end{aligned}
$$

Coming back to the previous notations i taking into account (54) and the definitions of $\mathbf{v}^{j, \delta, \delta}$ and $\mathbf{w}^{j, \varepsilon, \delta}$, we get from (70) for $l=2$

$$
\max _{0<t<T}\left\|\mathbf{u}^{j, \varepsilon}(\cdot, t)\right\|_{2, Q_{j}^{(\delta)}}^{(2)} \leq C_{1}(\delta), \quad j=f, s .
$$

Now we repeat all this with the function $\zeta^{(2 \delta)}$ and domain $\Omega^{(2 \delta)}: \zeta^{(2 \delta)}(\mathbf{x})=1$ for $\mathbf{x} \in \Omega^{(4 \delta)}$, and $\zeta^{(2 \delta)}(\mathbf{x})=0$ for $\mathbf{x} \in Q \backslash \Omega^{(28)}$. 
Namely, (71) implies for (55)

$$
\max _{0<t<T}\left\|\mathbf{F}^{\varepsilon, 2 \delta}(\cdot, t)\right\|_{2, \mathbb{R}^{2}}^{(1)}+\max _{0<t<T}\left\|\boldsymbol{\varphi}^{\varepsilon, \delta}(\cdot, t)\right\|_{2, \mathbb{R}^{2}}^{(2)} \leq C_{2}(\delta)
$$

and, consequently,

$$
\begin{aligned}
& \max _{0<t<T}\left\|\mathbf{v}^{j \varepsilon, 2 \delta}(\cdot, t)\right\|_{2, \mathbb{R}_{j}^{2}}^{(3)} \leq C_{3} \max _{0<t<T}\left\|\mathbf{w}^{j \varepsilon, 2 \delta}(\cdot, t)\right\|_{2, \mathbb{R}_{j}^{2}}^{(3)} \leq C_{3}(\delta), \quad j=f, s, \\
& \max _{0<t<T}\left\|\mathbf{u}^{j, \varepsilon}(\cdot, t)\right\|_{2, Q_{j}^{(2 \delta)}}^{(3)} \leq C_{4}(\delta), \quad j=f, s .
\end{aligned}
$$

The corresponding embedding theorem $W_{2}^{3}(\Omega) \rightarrow C^{1+\alpha}(\Omega)$ for $0<\alpha<1$ ([13], Theorem 2.1, p.61, Chapter 2)

$$
\max _{0<t<T}\left|\mathbf{u}^{j, \varepsilon}(\cdot, t)\right|_{Q_{j}^{(2 \delta)}}^{(1+\alpha)} \leq C \max _{0<t<T}\left\|\mathbf{u}^{j, \varepsilon}(\cdot, t)\right\|_{2, Q_{j}^{(2 \delta)}}^{(3)} \leq C_{5}(\delta), \quad j=f, s
$$

proves (52) and the statement of the lemma.

\subsection{Uniform bounds for density}

Let $\Gamma^{\varepsilon}(t) \subset Q_{f}$ be a smooth surface obtained by moving the initial position $\Gamma(0)$ along the trajectories of the velocity field $\mathbf{v}^{\varepsilon}$ :

$$
\frac{d \mathbf{x}}{d t}=\mathbf{v}^{\varepsilon}(\mathbf{x}, t), \quad \mathbf{x}(0)=\xi, \xi \in \Gamma(0)
$$

As we have mentioned above, the time $T$ is chosen from the condition

$$
\operatorname{dist}\left(\Gamma^{\varepsilon}(t), S^{ \pm}\right)>0
$$

Moreover, we suppose that

$$
\operatorname{dist}\left(\Gamma^{\varepsilon}(t), S^{ \pm}\right)>\varepsilon
$$

Lemma 6 Under the conditions of Theorem 2.1 let $\rho^{\varepsilon}$ be the solution of the problem (30). Then

$$
\max _{0<t<T}\left(\int_{Q_{f}^{(\delta)}}\left|\frac{\partial \rho^{\varepsilon}}{\partial t}(\mathbf{x}, t)\right| d x+\sum_{i=1}^{2} \int_{Q_{f}^{(\delta)}}\left|\frac{\partial \rho^{\varepsilon}}{\partial x_{i}}(\mathbf{x}, t)\right| d x\right) \leq C(\delta) .
$$

Proof Let $q_{i}=\frac{\partial \rho^{\varepsilon}}{\partial x_{i}}$.

Then

$$
\frac{\partial q_{i}}{\partial t}+\mathbf{v}^{\varepsilon} \cdot \nabla q_{i}=\sum_{j=1}^{n} a_{i j} q_{j}, \quad \mathbf{x} \in Q, 0<t<T,
$$

where $a_{i j}=-\frac{\partial v_{j}^{\varepsilon}}{\partial x_{i}}, i, j=1,2$, and

$$
\max _{0<t<T}\left|a_{i j}(\cdot, t)\right|_{2, Q_{j}^{(2 \delta)}}^{(1)} \leq C(\delta) .
$$


Note that $q_{i} \equiv 0$ near the boundaries $S^{ \pm}$, and $S^{0}$. This follows from the supposition on the behavior of the boundary $\Gamma^{\varepsilon}(t)$, and the choice of the time $T$.

Multiplying the equation for $q_{i}$ by $\frac{q_{i}}{\left(q_{i}^{2}+\delta^{2}\right)^{\frac{1}{2}}}$ and integrating by parts over $Q$ we arrive at the equality

$$
\frac{d}{d t} \int_{Q}\left(q_{i}^{2}+\delta^{2}\right)^{\frac{1}{2}} d x=-\int_{Q} \sum_{j=1}^{2} a_{i j} q_{j} \frac{q_{i}}{\left(q_{i}^{2}+\delta^{2}\right)^{\frac{1}{2}}} d x,
$$

and, consequently, the inequality

$$
\frac{d y}{d t} \leq C(\delta) y, \quad y(0) \leq C(\delta)
$$

for $y=\sum_{i=1}^{2} \int_{Q}\left(q_{i}^{2}+\delta^{2}\right)^{\frac{1}{2}} d x$.

The Gronwall inequality provides estimates (77) for $q_{i}, i=1,2$, and the transport equation (30) provides estimate (77) for the time derivative of $\rho^{\varepsilon}$.

Passage to non-smooth initial data, existence of a regular free boundary, existence of the maximal time interval and uniqueness of the solution are proved in the same way as in the previous papers [10] and [1].

\section{Acknowledgements}

The authors would like to thank the referee for his/her careful reading of the manuscript and the useful suggestions and comments.

\section{Funding}

The research has been supported by the Science Committee of the Ministry of Education and Science of the Republic of Kazakhstan, grant 0980/GF4.

\section{Competing interests}

The authors declare that they have no competing interests.

\section{Authors' contributions}

The authors have made equal contributions to each part of this article. All the authors read and approved the final manuscript.

\section{Author details}

${ }^{1}$ Yachay Tech University, Hacienda San Jose s/n y Proyecto Yachay, San Miguel de Urcuqui, Ecuador. ${ }^{2}$ Belgorod National Research University, Pobedi 85, Belgorod, 308015, Russia. ${ }^{3}$ Kazakh-British Technical University, Toli bi 59, Almaty, 050000, Kazakhstan.

\section{Publisher's Note}

Springer Nature remains neutral with regard to jurisdictional claims in published maps and institutional affiliations.

Received: 6 March 2017 Accepted: 18 May 2017 Published online: 06 June 2017

\section{References}

1. Meirmanov, A, Zimin, R, Shiyapov, K: The Muskat problem at the microscopic level for a single capillary. Bound. Value Probl. 2015, 71 (2015)

2. Antontsev, SN, Kazhikhov, AV, Monakhov, VN: Series: studies in mathematics and its applications. In: Boundary Value Problems in Mechanics of Nonhomogeneous Fluids, p. 308. North-Holland, Amsterdam (1990)

3. Cattabriga, L: Su un problema al contorno relativo al sistema di equazioni di Stokes. Rend. Semin. Mat. Univ. Padova 31, 308-340 (1961)

4. Giga, Y, Takahashi, S: On global weak solutions of nonstationary two-phase Stokes flow. SIAM J. Math. Anal. 25, 876-893 (1994)

5. Fernández-Cara, E, Guillén, F, Ortega, R: Some theoretical results for visco-plastic and dilatant fluids with variable density. Nonlinear Methods and Appl. 28, 1079-1100 (1997)

6. Nouri, A, Poupaud, F: An existence theorem for the multifluid Navier-Stokes problem. J. Differ. Equ. 13, 463-484 (1995) 
7. Simon, J: Nonhomogeneous viscous incompressible fluids: existence of velocity, density, and pressure. SIAM J. Math. Anal. 21, 1093-1117 (1990)

8. Yih, C-S: Dynamics of Nonhomogeneous Fluids. CollierMacmillan, London (1965)

9. Solonnikov, VA, Ladyzhenskaya, OA: On unique solvability of an initial-boundary value problem for viscous nonhomogeneous fluids. Zapiski Nauchn. Semin. LOMI 52, 52-109 (1975)

10. Antontsev, S, Meirmanov, A, Yurinsky, V: A free boundary problem for Stokes equations: classical solutions. Interfaces Free Bound. 2, 413-424 (2000)

11. Meirmanov, A: The muskat problem for viscoelastic filtration. Interfaces Free Bound. 122, 71-88 (2011)

12. Meirmanov, A: Mathematical Models for Poroelastic Flows. Atlantis Press, Amsterdam-Paris-Beijing (2014)

13. Ladyzhenskaya, OA, Solonnikov, VA, Ural'ceva, NN: Translations of mathematical monographs. In: Linear and Quasi-Linear Equations of Parabolic Type, p. 648. American Mathematical Society, New-York (1968)

14. Kirk, WA, Sims, B: Handbook of Metric Fixed Point Theory. Kluwer Academic, London (2001)

15. Zheng, W: On Friedrichs-Poincaré-type inequalities. J. Math. Anal. Appl. 304, 542-551 (2005)

16. Ladyzhenskaya, OA: The Mathematical Theory of Viscous Incompressible Flows. Gordon and Breach Science Publishers, New York (1969)

\section{Submit your manuscript to a SpringerOpen ${ }^{\ominus}$ journal and benefit from:}

- Convenient online submission

Rigorous peer review

- Immediate publication on acceptance

Open access: articles freely available online

- High visibility within the field

- Retaining the copyright to your article 